\title{
Presentations for rook partition monoids and algebras and their singular ideals
}

\author{
James East \\ Centre for Research in Mathematics; School of Computing, Engineering and Mathematics \\ University of Western Sydney, Locked Bag 1797, Penrith NSW 2751, Australia \\ J.East @ uws.edu.au
}

\begin{abstract}
We obtain several presentations by generators and relations for the rook partition monoids and algebras, as well as their singular ideals. Among other results, we also calculate the minimal sizes of generating sets (some of our presentations use such minimal-size generating sets), and show that the singular part of the rook partition monoid is generated by its idempotents.

Keywords: Partition algebra, Partition monoid, Rook partition algebra, Rook partition monoid, Singular ideal, Presentations, Rank, Idempotent rank.
\end{abstract}

MSC: 20M05; 20M20.

\section{Introduction}

Diagram algebras have diverse origins and applications. For example, see Brauer [8] on invariant theory; Jones [42] and Kauffman [44] on knot theory; Temperley and Lieb [73], Jones [43] and Martin [58] on statistical mechanics; and more. One unifying theme is that diagram algebras often occur as centraliser algebras of classical groups, leading to various interesting extensions of (classical) Schur-Weyl duality [74. For example, the Brauer algebras are related in this way to the orthogonal groups [8], and the partition algebras to symmetric groups [43, 58]. In a highly influential 2005 paper, Halverson and Ram [37] gave (among many other things) an account of Schur-Weyl duality in the case of the partition algebras, making crucial use of a tower of algebras that saw the partition algebras $\mathbb{C} A_{k}(n)$ embedded into $\mathbb{C} A_{k+1}(n)$ via an intermediate subalgebra:

$$
\cdots \hookrightarrow \mathbb{C} A_{k}(n) \hookrightarrow \mathbb{C} A_{k+\frac{1}{2}}(n) \subseteq \mathbb{C} A_{k+1}(n) \hookrightarrow \cdots .
$$

Halverson and Ram [37] attributed their understanding of the "existence and importance" of the algebras $\mathbb{C} A_{k+\frac{1}{2}}(n)$ to Cheryl Grood, who studied them in their own right in [33], where they were called rook partition algebras, and given their own diagrammatic interpretation (see Section 2.1 below for details); Grood also noted that these intermediate algebras were used in earlier work of Martin [57,59]. The reason for the name is due to a connection with the so-called rook monoids (and associated algebras and deformations) studied by Halverson, Solomon and others [12, 18, 32, 34, 36, 68,72]. As noted by Grood [33], Solomon's discovery [72] of a Schur-Weyl duality for rook monoid algebras (see also [50]) led to the investigation of a number of other "rook diagram algebras", such the rook Brauer algebras [35,60], Motzkin algebras [5] and more. Such studies, and other considerations often to do with representation theory and/or statistical mechanics, have led to the discovery and investigation of a great many other families of diagram algebras $[6,10,11,45,61,62,67,69]$. 
In this article, we continue the study of the rook partition algebras, with our goal being to derive presentations by generators and relations. This continues a theme initiated by the current author in [20], where such presentations were obtained for the partition algebras themselves. Presentations are extremely useful tools for algebraists: for one thing, they allow representations (homomorphisms into other algebraic structures) to be defined by specifying the images of the generators and checking that the relations are preserved; this is especially helpful when the algebra in question is as complicated as the partition algebra. With respect to the partition algebras in particular, we refer to the recent work of Enyang on Jucys-Murphy elements [25] and seminormal forms [26], in which the presentations from [20,37] played a crucial role. Presentations also feature heavily in the work of Lehrer and Zhang on diagram categories and invariant theory [53, 54].

Key to the approach used in [20] was the observation of Wilcox [75] (also implicit in [37]) that diagram algebras (including the partition algebras) arise as twisted semigroup algebras of corresponding diagram semigroups. This allows one to obtain information (concerning cellularity [17, 24, 31, 75] or presentations [20,21], for example) about the algebras from corresponding information about the associated semigroups. Conversely, the theory of diagram algebras has led to a number of important families of semigroups and monoids that have been studied with increasing vigour in recent years; see for example [7, 13 17, 20, 21, 23, 24, 28, 49, 51, 56, 63, 64, 71], and especially the work of Auinger and his collaborators [1,4] on equational theories of involution semigroups. A number of other authors have studied presentations of diagram semigroups [7, 49, 56, 71]. We hope that the techniques we introduce here to deal with the more complicated rook partition algebras will prove useful in other investigations: for example, in the context of quasi-partition algebras [11] or coloured partition algebras [6, 69] and their rook versions [46].

As stated above, our focus in this article is on the rook partition monoid and algebra, $\mathcal{R P}_{n}$ and $F^{\tau}\left[\mathcal{R} \mathcal{P}_{n}\right]$, and their singular ideals, $\mathcal{R} \mathcal{P}_{n} \backslash \mathcal{S}_{n}$ and $F^{\tau}\left[\mathcal{R} \mathcal{P}_{n} \backslash \mathcal{S}_{n}\right]$; see Sections 2 and 5 for precise definitions. In particular, we obtain a number of presentations for each of these algebraic systems (Theorems 3.14, 4.7, 4.11, 4.12 and 5.1); among other results, we calculate the smallest sizes of generating sets (Theorems 3.15 and 4.14), and show that $\mathcal{R} \mathcal{P}_{n} \backslash \mathcal{S}_{n}$ is generated by its idempotents (Proposition 3.1 and Remark 3.2). Our approach is quite different to previous studies of (singular) diagram semigroups and algebras [7,20,21,49, 56, 71], in the sense that we first obtain a presentation for the singular rook partition monoid $\mathcal{R} \mathcal{P}_{n} \backslash \mathcal{S}_{n}$ (the subject of Section 3), and then use this to bootstrap up to a number of presentations for the (full) rook partition monoid $\mathcal{R} \mathcal{P}_{n}$ (in Section 4). (Although this appears to be the first instance of such a "singular first" approach, we note that a somewhat similar method was used in the author's recent work on (singular) symmetric inverse semigroups in [22].) Our approach makes crucial use of the author's presentations for the (ordinary) partition monoid $\mathcal{P}_{n}$ [20] and its singular ideal $\mathcal{P}_{n} \backslash \mathcal{S}_{n}$ [21]; these are stated in Section 2, along with various definitions, notations, background information, and illustrative examples. Finally, in Section [5, we apply general results on twisted semigroup algebras from [20] to obtain (algebra) presentations for the rook partition algebra $F^{\tau}\left[\mathcal{R} \mathcal{P}_{n}\right]$ and its singular ideal $F^{\tau}\left[\mathcal{R} \mathcal{P}_{n} \backslash \mathcal{S}_{n}\right]$; see Theorem $[5.1$.

\section{Preliminaries}

In this section, we gather the preliminary material we will need in our investigations. In particular, we define the rook partition monoids, identify several key submonoids, develop the notation and parameters needed to formulate and prove our results, and review previous results on (ordinary) partition monoids that will play a role in our study. (We postpone the definition of the rook partition algebra until Section 5.) 


\subsection{The rook partition monoid}

We adapt the treatment of Grood [33]. Fix a non-negative integer $n$, and write $[n]=\{1, \ldots, n\}$ and $[n]^{\prime}=\left\{1^{\prime}, \ldots, n^{\prime}\right\}$. By a rook partition of degree $n$, we mean a set partition of a subset of $[n] \cup[n]^{\prime}$ : i.e., a collection $\alpha=\left\{A_{i}: i \in I\right\}$, for some (possibly empty) indexing set $I$, where the $A_{i}$ are pairwise disjoint non-empty subsets of $[n] \cup[n]^{\prime}$; the $A_{i}$ are called the blocks of $\alpha$. We write $\operatorname{supp}(\alpha)=\bigcup_{i \in I} A_{i}$, and call this the support of $\alpha$. The elements of $\left([n] \cup[n]^{\prime}\right) \backslash \operatorname{supp}(\alpha)$ are called the rook dots of $\alpha$. We write $\mathcal{R} \mathcal{P}_{n}$ for the set of all rook partitions of degree $n$. For example, the rook partition

$$
\alpha=\left\{\left\{1,2,4,3^{\prime}\right\},\left\{5,6,4^{\prime}, 5^{\prime}\right\},\left\{7,8,8^{\prime}\right\},\left\{2^{\prime}, 6^{\prime}, 7^{\prime}\right\},\left\{9^{\prime}, 10^{\prime}\right\}\right\} \in \mathcal{R} \mathcal{P}_{10}
$$

has support $\left\{1,2,4,5,6,7,8,2^{\prime}, 3^{\prime}, 4^{\prime}, 5^{\prime}, 6^{\prime}, 7^{\prime}, 8^{\prime}, 9^{\prime}, 10^{\prime}\right\}$, and rook dots $3,9,10,1^{\prime}$.

We may represent an element $\alpha \in \mathcal{R} \mathcal{P}_{n}$ graphically as follows. We arrange vertices $1, \ldots, n$ in a horizontal row (increasing from left to right) with vertices $1^{\prime}, \ldots, n^{\prime}$ directly below. We colour each vertex from $\operatorname{supp}(\alpha)$ black, and each rook dot white, and add edges between black vertices in such a way that two vertices are joined by a path if and only if they belong to the same block of $\alpha$. Such a graph is called a rook diagram of $\alpha$. The rook partition $\alpha \in \mathcal{R} \mathcal{P}_{10}$ defined at the end of the previous paragraph is pictured in Figure 1 (top left). The graphical representation of a rook partition is not unique, but, as in [33], we regard two rook diagrams as equivalent if they have the same rook dots and the same connected components. Generally, we identify a rook partition $\alpha \in \mathcal{R} \mathcal{P}_{n}$ with any rook diagram representing it.

To describe the product in $\mathcal{R} \mathcal{P}_{n}$, consider two rook partitions $\alpha, \beta \in \mathcal{R} \mathcal{P}_{n}$. Write $[n]^{\prime \prime}=\left\{1^{\prime \prime}, \ldots, n^{\prime \prime}\right\}$. Let $\alpha_{\vee}$ be the graph obtained from $\alpha$ by changing the label of each lower vertex $i^{\prime}$ to $i^{\prime \prime}$. Similarly, let $\beta^{\wedge}$ be the graph obtained from $\beta$ by changing the label of each upper vertex $i$ to $i^{\prime \prime}$. Consider now the graph $\Gamma(\alpha, \beta)$ on the vertex set $[n] \cup[n]^{\prime} \cup[n]^{\prime \prime}$ obtained by joining $\alpha_{\vee}$ and $\beta^{\wedge}$ together so that each lower vertex $i^{\prime \prime}$ of $\alpha_{\vee}$ is identified with the corresponding upper vertex $i^{\prime \prime}$ of $\beta^{\wedge}$, and colouring a vertex $i^{\prime \prime}$ white in $\Gamma(\alpha, \beta)$ if it is white in either $\alpha_{\vee}$ or $\beta^{\wedge}$. We call $\Gamma(\alpha, \beta)$ the product graph of $\alpha, \beta$. We now let $\alpha \beta \in \mathcal{R} \mathcal{P}_{n}$ be the (rook partition corresponding to any) rook diagram such that:

- $\alpha \beta$ has a rook dot $x \in[n] \cup[n]^{\prime}$ if and only if $x$ is connected by a (possibly empty) path to a white vertex in $\Gamma(\alpha, \beta)$, and

- two black vertices $x, y \in[n] \cup[n]^{\prime}$ are connected by a path in $\alpha \beta$ if and only if they are connected by a path in $\Gamma(\alpha, \beta)$.

An example calculation (with $n=10$ ) is carried out in Figure 1.

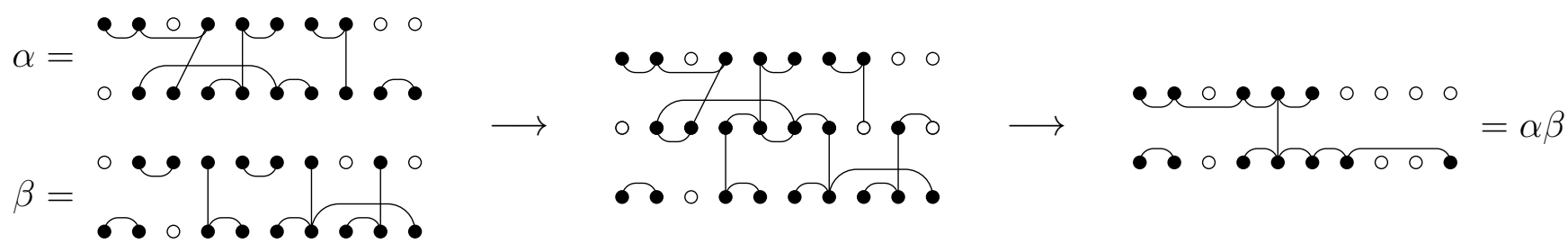

Figure 1: Two rook partitions $\alpha, \beta \in \mathcal{R} \mathcal{P}_{10}$ (left), the product $\alpha \beta \in \mathcal{R P}_{10}$ (right), and the product graph $\Gamma(\alpha, \beta)$ (centre).

This operation is associative, and gives $\mathcal{R} \mathcal{P}_{n}$ the structure of a monoid; the identity element is

$$
\left.1=\left\{\left\{1,1^{\prime}\right\}, \ldots,\left\{n, n^{\prime}\right\}\right\}=\bullet_{\bullet}^{1} \cdots\right\rfloor^{n} \in \mathcal{R} \mathcal{P}_{n}
$$

Before we can say more about the structure of $\mathcal{R} \mathcal{P}_{n}$, we first introduce some notation. Let $\alpha \in \mathcal{R} \mathcal{P}_{n}$. We call a block of $\alpha$ a transversal block if it has non-empty intersection with both $[n]$ and $[n]^{\prime}$, and 
a non-transversal block otherwise; we will also distinguish between upper and lower non-transversal blocks (defined in an obvious way). The rank of $\alpha$, denoted $\operatorname{rank}(\alpha)$, is defined to be the number of transversal blocks of $\alpha$. Note that $0 \leq \operatorname{rank}(\alpha) \leq n$. For $x \in \operatorname{supp}(\alpha)$, we write $[x]_{\alpha}$ for the block of $\alpha$ containing $x$; we also define $[x]_{\alpha}=\{x\}$ if $x$ is a rook dot of $\alpha$. We then define the domain and codomain of $\alpha$ to be the sets

$$
\operatorname{dom}(\alpha)=\left\{x \in[n]:[x]_{\alpha} \cap[n]^{\prime} \neq \emptyset\right\} \quad \text { and } \quad \operatorname{codom}(\alpha)=\left\{x \in[n]:\left[x^{\prime}\right]_{\alpha} \cap[n] \neq \emptyset\right\} .
$$

We also define the kernel and cokernel of $\alpha$ to be the equivalences

$$
\operatorname{ker}(\alpha)=\left\{(x, y) \in[n] \times[n]:[x]_{\alpha}=[y]_{\alpha}\right\} \quad \text { and } \quad \operatorname{coker}(\alpha)=\left\{(x, y) \in[n] \times[n]:\left[x^{\prime}\right]_{\alpha}=\left[y^{\prime}\right]_{\alpha}\right\} \text {. }
$$

For example, with $\alpha \in \mathcal{R} \mathcal{P}_{10}$ as in Figure 1 (top left),

$$
\begin{gathered}
\operatorname{rank}(\alpha)=3, \quad \operatorname{dom}(\alpha)=\{1,2,4,5,6,7,8\}, \quad \operatorname{codom}(\alpha)=\{3,4,5,8\}, \\
\operatorname{ker}(\alpha)=(1,2,4|3| 5,6|7,8| 9 \mid 10), \quad \operatorname{coker}(\alpha)=(1|2,6,7| 3|4,5| 8 \mid 9,10),
\end{gathered}
$$

using an obvious notation for equivalences.

It is immediate from the definitions that the following hold for all $\alpha, \beta \in \mathcal{R} \mathcal{P}_{n}$ :

$$
\operatorname{dom}(\alpha \beta) \subseteq \operatorname{dom}(\alpha), \quad \operatorname{ker}(\alpha \beta) \supseteq \operatorname{ker}(\alpha), \quad \operatorname{codom}(\alpha \beta) \subseteq \operatorname{codom}(\beta), \quad \operatorname{coker}(\alpha \beta) \supseteq \operatorname{coker}(\beta) .
$$

We write $\Delta=\{(x, x): x \in[n]\}$ for the trivial relation on $[n]$ : i.e., the equality relation. The rook partition monoid $\mathcal{R} \mathcal{P}_{n}$ contains a number of important submonoids, defined as follows:

- $\mathcal{P}_{n}=\left\{\alpha \in \mathcal{R} \mathcal{P}_{n}: \operatorname{supp}(\alpha)=[n] \cup[n]^{\prime}\right\}$, the partition monoid [20, 37];

- $\mathcal{I}_{n}=\left\{\alpha \in \mathcal{P}_{n}: \operatorname{ker}(\alpha)=\operatorname{coker}(\alpha)=\Delta\right\}$, the symmetric inverse monoid [52,55];

- $\mathcal{J}_{n}=\left\{\alpha \in \mathcal{P}_{n}: \operatorname{dom}(\alpha)=\operatorname{codom}(\alpha)=[n]\right\}$, the dual symmetric inverse monoid [29];

- $\mathcal{S}_{n}=\left\{\alpha \in \mathcal{R} \mathcal{P}_{n}: \operatorname{rank}(\alpha)=n\right\}$, the symmetric group [9]; and

- $\mathcal{R}_{n}=\left\{\alpha \in \mathcal{R P}_{n}: \operatorname{ker}(\alpha)=\operatorname{coker}(\alpha)=\Delta, \operatorname{supp}(\alpha)=\operatorname{dom}(\alpha) \cup \operatorname{codom}(\alpha)^{\prime}\right\}$, the rook monoid [32,72].

Elements of these sumbonoids are pictured in Figure 2, along with the various containments among the submonoids.
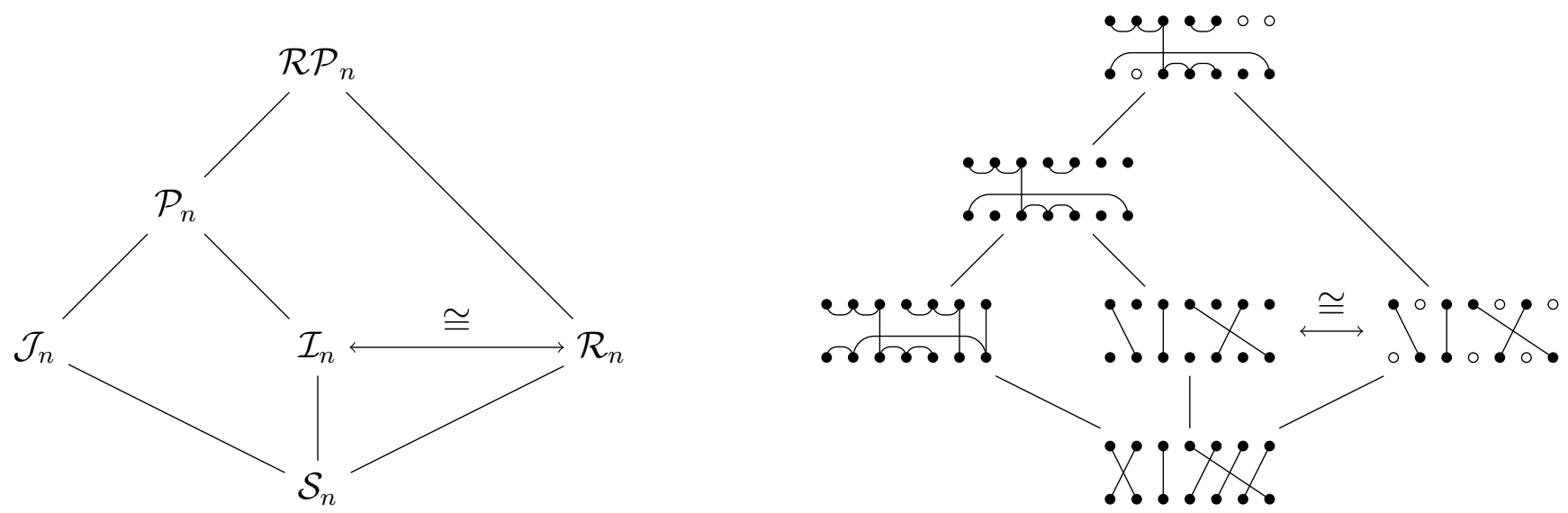

Figure 2: Important submonoids of $\mathcal{R} \mathcal{P}_{n}$ (left) and representative elements from each submonoid (right).

Note that $\mathcal{I}_{n}$ and $\mathcal{R}_{n}$ are isomorphic (via an obvious map $\mathcal{I}_{n} \rightarrow \mathcal{R}_{n}$ that colours all non-transversal blocks of $\alpha \in \mathcal{I}_{n}$ white), and that $\mathcal{S}_{n}$ is the group of units of $\mathcal{R} \mathcal{P}_{n}$ (in fact, the group of units of all the 
stated submonoids). It follows from [20, Theorem 32] that $\mathcal{P}_{n}$ is equal to the join $\mathcal{I}_{n} \vee \mathcal{J}_{n}=\left\langle\mathcal{I}_{n} \cup \mathcal{J}_{n}\right\rangle$. The join $\mathcal{J}_{n} \vee \mathcal{R}_{n}$ is the partial dual symmetric inverse monoid studied in [47, 48. The join $\mathcal{I}_{n} \vee \mathcal{R}_{n}$ is a "rook version" of the symmetric inverse monoid; to the author's knowledge, it has not been explicitly studied in the literature. As noted in [33], $\mathcal{R} \mathcal{P}_{n}$ is isomorphic to the submonoid of $\mathcal{P}_{n+1}$ consisting of all (ordinary) partitions of degree $n+1$ such that $n+1$ and $(n+1)^{\prime}$ belong to the same block; these submonoids were introduced in [57, 59] and played a central role in [37].

We now describe a convenient notation for rook partitions, extending the notation introduced for ordinary partitions in [23]. With this in mind, let $\alpha \in \mathcal{R} \mathcal{P}_{n}$. We write

$$
\alpha=\left(\begin{array}{c|c|c|c|c|c||c}
A_{1} & \cdots & A_{r} & C_{1} & \cdots & C_{p} & P \\
B_{1} & \cdots & B_{r} & D_{1} & \cdots & D_{q} & Q
\end{array}\right)
$$

to indicate that $\alpha$ has (writing $A^{\prime}=\left\{a^{\prime}: a \in A\right\}$ for $A \subseteq[n]$ ):

- transversal blocks $A_{i} \cup B_{i}^{\prime}$, for each $1 \leq i \leq r$,

- upper non-transversal blocks $C_{i}$, for each $1 \leq i \leq p$,

- lower non-transversal blocks $D_{i}^{\prime}$, for each $1 \leq i \leq q$, and

- rook dots $x$, for each $x \in P \cup Q^{\prime}$.

So, for example, with $\alpha \in \mathcal{R} \mathcal{P}_{10}$ as in Figure 1 (top left), we have

$$
\alpha=\left(\begin{array}{c|c|c|c|c||c}
1,2,4 & 5,6 & 7,8 & \multicolumn{2}{|l||}{3,9,10} \\
\cline { 3 - 5 } 3 & 4,5 & 8 & 2,6,7 & 9,10 & 1
\end{array}\right) .
$$

In the above notation, it is possible that any of $r, p, q,|P|,|Q|$ could be 0 , in which case we may use simplified versions of the above notation. If $\alpha$ has no rook dots (so that $\alpha \in \mathcal{P}_{n}$ ), we will omit $P$ and $Q$, and write

$$
\alpha=\left(\begin{array}{c|c|c|c|c|c}
A_{1} & \cdots & A_{r} & C_{1} & \cdots & C_{p} \\
\cline { 2 - 5 } B_{1} & \cdots & B_{r} & D_{1} & \cdots & D_{q}
\end{array}\right)
$$

as in [23]. Similarly, if $\alpha$ has no rook dots and no non-transversal blocks (so that $\alpha \in \mathcal{J}_{n}$ ), then we will simply write

$$
\alpha=\left(\begin{array}{c|c|c}
A_{1} & \cdots & A_{r} \\
B_{1} & \cdots & B_{r}
\end{array}\right) .
$$

If $\alpha \in \mathcal{I}_{n}($ so $\operatorname{ker}(\alpha)=\operatorname{coker}(\alpha)=\Delta$ and $\alpha$ has no rook dots), we will write

$$
\alpha=\left(\begin{array}{c|c|c}
a_{1} & \cdots & a_{r} \\
b_{1} & \cdots & b_{r}
\end{array}\right)
$$

to indicate that the transversal blocks of $\alpha$ are $\left\{a_{i}, b_{i}^{\prime}\right\}$ (for each $1 \leq i \leq r$ ) and that every other block is a (non-rook) singleton. We will use other simplifications from time to time, but it will always be clear what is meant.

Finally, we note that $\mathcal{R} \mathcal{P}_{n}$ has a natural (anti-)involution ${ }^{*}: \mathcal{R} \mathcal{P}_{n} \rightarrow \mathcal{R} \mathcal{P}_{n}: \alpha \mapsto \alpha^{*}$, defined by

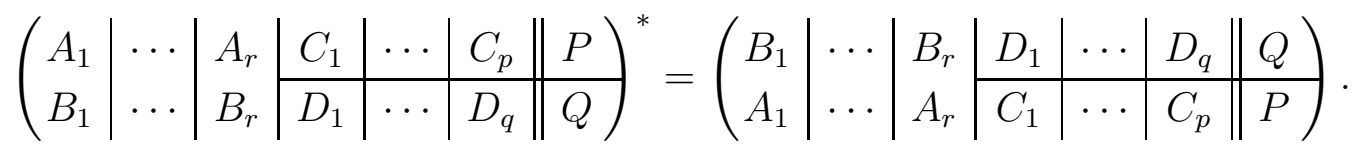

Diagrammatically, $\alpha^{*}$ is obtained by turning (a graphical representation of) $\alpha$ upside-down. This involution reflects the structure of $\mathcal{R} \mathcal{P}_{n}$ as a regular $*$-semigroup (as defined in [66]). That is, the 
following hold for all $\alpha, \beta \in \mathcal{R} \mathcal{P}_{n}$ (as may easily be checked diagrammatically, or follows from the above-mentioned embedding $\left.\mathcal{R} \mathcal{P}_{n} \rightarrow \mathcal{P}_{n+1}\right)$ :

$$
\left(\alpha^{*}\right)^{*}=\alpha, \quad(\alpha \beta)^{*}=\beta^{*} \alpha^{*}, \quad \alpha \alpha^{*} \alpha=\alpha, \quad \alpha^{*} \alpha \alpha^{*}=\alpha^{*} .
$$

This $*$-regular structure leads to a duality that will help simplify several proofs, and has played a very important role in many other studies of diagram monoids; see [1, 3, 20, 21, 23, 24], among many others.

We conclude this section with two structural results, concerning normal forms for $\mathcal{P}_{n}$ (Proposition 2.1) and $\mathcal{R} \mathcal{P}_{n}$ (Proposition 2.3). They will both be useful on a number of occasions. The first of these gives a convenient factorisation for the elements of $\mathcal{P}_{n}$ that was used in the proof of [23, Theorem 30]; its proof is easy and is omitted.

Proposition 2.1. Let $\alpha \in \mathcal{P}_{n}$, and write

$$
\alpha=\left(\begin{array}{l|l|l|l|l|l}
A_{1} & \cdots & A_{r} & C_{1} & \cdots & C_{p} \\
\cline { 2 - 4 } B_{1} & \cdots & B_{r} & D_{1} & \cdots & D_{q}
\end{array}\right) .
$$

For each $i \in[r]$, choose some $a_{i} \in A_{i}$ and $b_{i} \in B_{i}$. Then $\alpha=\beta \gamma \delta$, where

$$
\beta=\left(\begin{array}{c|c|c|c|c|c}
A_{1} & \ldots & A_{r} & C_{1} & \ldots & C_{p} \\
A_{1} & \ldots & A_{r} & C_{1} & \ldots & C_{p}
\end{array}\right), \quad \gamma=\left(\begin{array}{c|c|c}
a_{1} & \ldots & a_{r} \\
b_{1} & \ldots & b_{r}
\end{array}\right), \quad \delta=\left(\begin{array}{c|c|c|c|c|c}
B_{1} & \ldots & B_{r} & D_{1} & \ldots & D_{q} \\
B_{1} & \ldots & B_{r} & D_{1} & \ldots & D_{q}
\end{array}\right) .
$$

Remark 2.2. Since $\beta, \delta \in \mathcal{J}_{n}$ and $\gamma \in \mathcal{I}_{n}$ (as defined above), Proposition 2.1 gives another proof of the above-mentioned fact that $\mathcal{P}_{n}=\mathcal{I}_{n} \vee \mathcal{J}_{n}=\left\langle\mathcal{I}_{n} \cup \mathcal{J}_{n}\right\rangle$.

As an example to illustrate Proposition 2.1, consider the partition

$$
\alpha=\left\{\left\{1,2,4,3^{\prime}\right\},\left\{5,6,4^{\prime}, 5^{\prime}\right\},\left\{7,8,8^{\prime}\right\},\{3\},\{9,10\},\left\{1^{\prime}\right\},\left\{2^{\prime}, 6^{\prime}, 7^{\prime}\right\},\left\{9^{\prime}, 10^{\prime}\right\}\right\} \in \mathcal{P}_{10} .
$$

Its factorisation $\alpha=\beta \gamma \delta$ (for some choice of the $a_{i}, b_{i}$ ), as in Proposition 2.1, is shown in Figure 3 ,

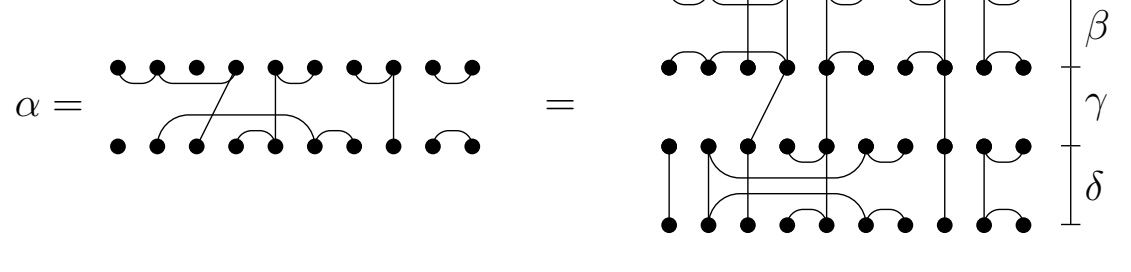

Figure 3: An illustration of the factorisation $\alpha=\beta \gamma \delta$ from Proposition 2.1, where $\alpha \in \mathcal{P}_{10}$.

Consider a subset $A \subseteq[n]$, and write $A^{c}=\left\{i_{1}, \ldots, i_{r}\right\}$. Here are elsewhere, we will write $A^{c}$ for the complement $[n] \backslash A$. We define

$$
\bar{o}_{A}=\left(\begin{array}{l|l|l||l}
i_{1} & \cdots & i_{r} & A \\
& \cdots & i_{r} & A
\end{array}\right)
$$

to be the (unique) element of $\mathcal{R} \mathcal{P}_{n}$ with rook dots $x$ for each $x \in A \cup A^{\prime}$, and transversal blocks $\left\{j, j^{\prime}\right\}$ for each $j \in A^{c}$. (The reason for the (over-line) notation will become clear shortly.) So, for example, with $n=10$ and $A=\{2,4,7,8,10\}$,

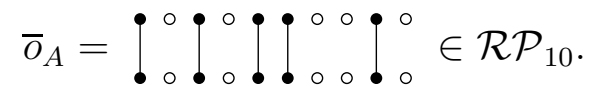

To simplify the statement of the next result, we will allow ourselves to refer to the empty set $\emptyset$ as a block of any element of $\mathcal{P}_{n}$. 
Proposition 2.3. For any rook partition $\alpha \in \mathcal{R} \mathcal{P}_{n}, \alpha=\bar{o}_{P} \beta \bar{o}_{Q}$ for unique (possibly empty) subsets $P, Q \subseteq[n]$ and a unique partition $\beta \in \mathcal{P}_{n}$ such that $P$ and $Q^{\prime}$ are (possibly empty) blocks of $\beta$. Moreover, if $\alpha \in \mathcal{R} \mathcal{P}_{n} \backslash \mathcal{S}_{n}$, then $\beta \in \mathcal{P}_{n} \backslash \mathcal{S}_{n}$.

Proof. Write

$$
\alpha=\left(\begin{array}{c|c|c|c|c|c||c}
A_{1} & \cdots & A_{r} & C_{1} & \cdots & C_{p} & P \\
\cline { 2 - 5 } & \cdots & B_{r} & D_{1} & \cdots & D_{q} & Q
\end{array}\right) .
$$

Let $\beta \in \mathcal{P}_{n}$ be the partition obtained by replacing the rook dots from $\alpha$ with the (possibly empty) blocks $P$ and $Q^{\prime}$. It is then clear that $\alpha=\bar{o}_{P} \beta \bar{o}_{Q}$. This establishes the existence part of the result. For the uniqueness part, suppose that $\alpha=\bar{o}_{A} \gamma \bar{o}_{B}$ where $A$ and $B^{\prime}$ are (possibly empty) blocks of $\gamma$. The rook dots of $\bar{o}_{A} \gamma \bar{o}_{B}$ are clearly the elements of $A \cup B^{\prime}$. The blocks of $\gamma$ other than $A$ and $B^{\prime}$ also clearly coincide with the (non-rook) blocks of $\bar{o}_{A} \gamma \bar{o}_{B}$. This all shows that $A=P, B=Q$ and $\gamma=\beta$.

Finally, suppose $\alpha \in \mathcal{R} \mathcal{P}_{n} \backslash \mathcal{S}_{n}$. If $\alpha$ has a rook dot, then $\beta$ has a non-transversal block. If $\alpha$ has no rook dots, then $\beta=\alpha$. So in either case, $\beta \in \mathcal{P}_{n} \backslash \mathcal{S}_{n}$.

Remark 2.4. An alternative factorisation $\alpha=\bar{o}_{P} \beta \bar{o}_{Q}$ could be proved, with $P \cup Q^{\prime}$ a (possibly empty) block of $\beta$ (instead of separate blocks $P, Q^{\prime}$ ). However, the implication $\alpha \in \mathcal{R} \mathcal{P}_{n} \backslash \mathcal{S}_{n} \Rightarrow \beta \in \mathcal{P}_{n} \backslash \mathcal{S}_{n}$ would not hold in general: namely, if $\alpha \in \mathcal{R}_{n}$ and $\operatorname{rank}(\alpha)=n-1$, then $\beta \in \mathcal{S}_{n}$.

As an example to illustrate Proposition 2.3, consider (once again) the rook partition

$$
\alpha=\left\{\left\{1,2,4,3^{\prime}\right\},\left\{5,6,4^{\prime}, 5^{\prime}\right\},\left\{7,8,8^{\prime}\right\},\left\{2^{\prime}, 6^{\prime}, 7^{\prime}\right\},\left\{9^{\prime}, 10^{\prime}\right\}\right\} \in \mathcal{R} \mathcal{P}_{10}
$$

Its factorisation $\alpha=\bar{o}_{P} \beta \bar{o}_{Q}$, as in Proposition 2.3, is shown in Figure 4.

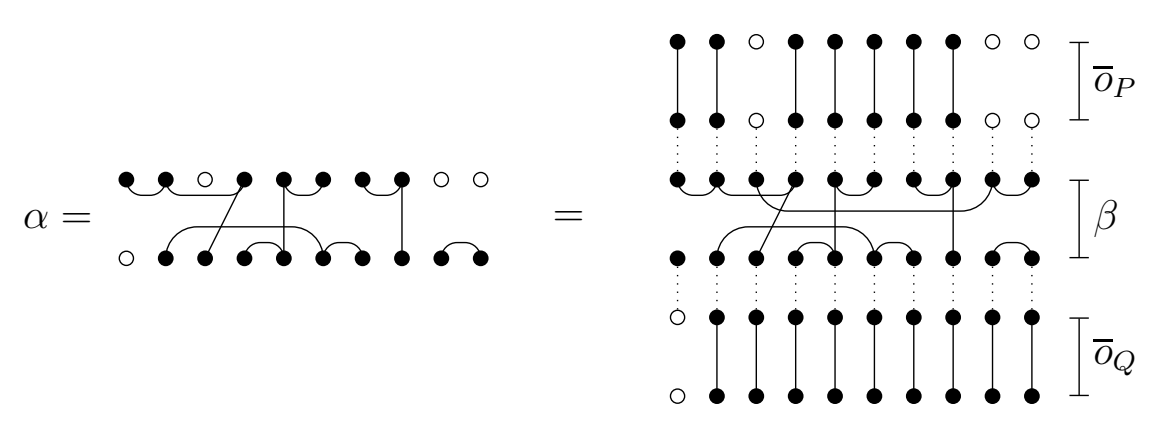

Figure 4: An illustration of the factorisation $\alpha=\bar{o}_{P} \beta \bar{o}_{Q}$ from Proposition $\left[2.3\right.$, where $\alpha \in \mathcal{R} \mathcal{P}_{10}$.

\subsection{Semigroups and presentations}

We will be dealing extensively with both semigroup and monoid presentations, so we now take the time to fix our notation for these, as well as some general semigroup notions. For further background on semigroups, the reader is referred to a monograph such as [38] or [41].

An equivalence relation $\sim$ on a semigroup $S$ is a congruence if $a \sim b$ and $c \sim d$ together imply $a c \sim b d$, for all $a, b, c, d \in S$. If $\sim$ is a congruence on $S$, then the quotient $S / \sim$, which consists of all $\sim$-classes of $S$, is a semigroup under the natural induced operation. The fundamental homomorphism theorem (for semigroups) states that if $\phi: S \rightarrow T$ is a semigroup homomorphism, then $S / \operatorname{ker}(\phi) \cong \operatorname{im}(\phi)$, where $\operatorname{ker}(\phi)$ is the congruence $\{(a, b) \in S \times S: a \phi=b \phi\}$.

Let $X$ be an alphabet, and denote by $X^{+}$(resp., $X^{*}$ ) the free semigroup (resp., free monoid) on $X$. If $R \subseteq X^{+} \times X^{+}$(resp., $R \subseteq X^{*} \times X^{*}$ ), we denote by $R^{\sharp}$ the congruence on $X^{+}$(resp., $X^{*}$ ) generated 
by $R$. We say a semigroup (resp., monoid) $S$ has semigroup (resp., monoid) presentation $\langle X: R\rangle$ if $S \cong X^{+} / R^{\sharp}$ (resp., $S \cong X^{*} / R^{\sharp}$ ) or, equivalently, if there is an epimorphism $X^{+} \rightarrow S$ (resp., $X^{*} \rightarrow S$ ) with kernel $R^{\sharp}$. If $\phi$ is such an epimorphism, we say $S$ has presentation $\langle X: R\rangle$ via $\phi$. A relation $\left(w_{1}, w_{2}\right) \in R$ will usually be displayed as an equation: $w_{1}=w_{2}$. We will always be careful to specify whether a given presentation is a semigroup or monoid presentation.

We denote the empty word (over any alphabet) by 1 (so $X^{*} \backslash X^{+}=\{1\}$ for any alphabet $X$ ). If $w=x_{1} \cdots x_{k}$, where $x_{1}, \ldots, x_{k} \in X$, we write $\ell(w)=k$ for the length of $w$. The word $x_{i} \cdots x_{j}$ is considered to be empty if either:

(i) $i>j$ and the subscripts are understood to be increasing; or

(ii) $i<j$ and the subscripts are understood to be decreasing.

When we are dealing with semigroup presentations, such a word will always be a subword of a larger (nonempty) word.

\subsection{Presentations for $\mathcal{P}_{n} \backslash \mathcal{S}_{n}$ and $\mathcal{P}_{n}$}

In Sections 3 and 4 , we will give presentations for $\mathcal{R} \mathcal{P}_{n} \backslash \mathcal{S}_{n}$ and $\mathcal{R} \mathcal{P}_{n}$ (respectively). To do this, it is crucial to know presentations for $\mathcal{P}_{n} \backslash \mathcal{S}_{n}$ and $\mathcal{P}_{n}$, and we describe such presentations (from [20, 21]) in this section. Consider alphabets $E=\left\{e_{1}, \ldots, e_{n}\right\}$ and $T=\left\{t_{i j}: 1 \leq i<j \leq n\right\}$, and define a (semigroup) homomorphism

$$
\phi:(E \cup T)^{+} \rightarrow \mathcal{P}_{n} \backslash \mathcal{S}_{n}
$$

by

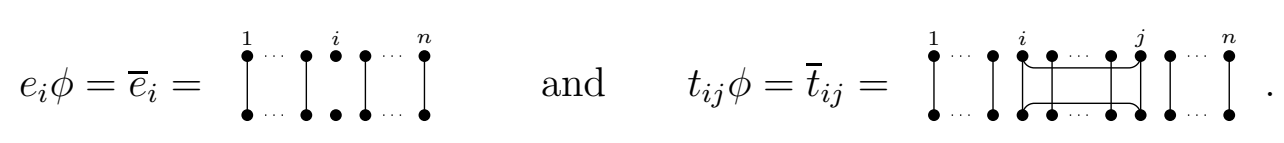

We will use symmetric notation when referring to the letters from $T$, so we write $t_{i j}=t_{j i}$ for all $1 \leq i<j \leq n$. Consider the relations

$$
\begin{aligned}
e_{i}^{2} & =e_{i} & & \text { for all } i \\
e_{i} e_{j} & =e_{j} e_{i} & & \text { for distinct } i, j \\
t_{i j}^{2} & =t_{i j} & & \text { for all } i, j \\
t_{i j} t_{k l} & =t_{k l} t_{i j} & & \text { for all } i, j, k, l \\
t_{i j} t_{j k} & =t_{j k} t_{k i} & & \text { for distinct } i, j, k \\
t_{i j} e_{k} & =e_{k} t_{i j} & & \text { if } k \notin\{i, j\} \\
t_{i j} e_{k} t_{i j} & =t_{i j} & & \text { if } k \in\{i, j\} \\
e_{k} t_{i j} e_{k} & =e_{k} & & \text { if } k \in\{i, j\} \\
e_{k} t_{k i} e_{i} t_{i j} e_{j} t_{j k} e_{k} & =e_{k} t_{k j} e_{j} t_{j i} e_{i} t_{i k} e_{k} & & \text { for distinct } i, j, k \\
e_{k} t_{k i} e_{i} t_{i j} e_{j} t_{j l} e_{l} t_{l k} e_{k} & =e_{k} t_{k l} e_{l} t_{l i} e_{i} t_{i j} e_{j} t_{j k} e_{k} & & \text { for distinct } i, j, k, l .
\end{aligned}
$$

The following is [21, Theorem 46].

Theorem 2.5. The singular part of the partition monoid, $\mathcal{P}_{n} \backslash \mathcal{S}_{n}$, has semigroup presentation

$$
\langle E \cup T:(\mathrm{R} 1-\mathrm{R} 10)\rangle,
$$

via $\phi$. 
Now consider the alphabets $S=\left\{s_{1}, \ldots, s_{n-1}\right\}, E=\left\{e_{1}, \ldots, e_{n}\right\}, Q=\left\{q_{1}, \ldots, q_{n-1}\right\}$, and define a (monoid) homomorphism

$$
\psi:(S \cup E \cup Q)^{*} \rightarrow \mathcal{P}_{n}
$$

by

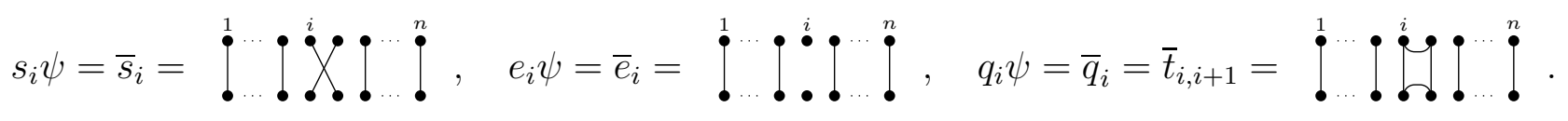

Consider the relations

$$
\begin{aligned}
s_{i}^{2} & =1 & & \text { for all } i \\
s_{i} s_{j} & =s_{j} s_{i} & & \text { if }|i-j|>1 \\
s_{i} s_{j} s_{i} & =s_{j} s_{i} s_{j} & & \text { if }|i-j|=1 \\
e_{i}^{2} & =e_{i} & & \text { for all } i \\
e_{i} e_{j} & =e_{j} e_{i} & & \text { for distinct } i, j \\
s_{i} e_{j} & =e_{j} s_{i} & & \text { if } j \notin\{i, i+1\} \\
s_{i} e_{i} & =e_{i+1} s_{i} & & \text { for all } i \\
e_{i} e_{i+1} s_{i} & =e_{i} e_{i+1} & & \text { for all } i \\
q_{i}^{2} & =q_{i} & & \text { for all } i \\
q_{i} q_{j} & =q_{j} q_{i} & & \text { for distinct } i, j \\
s_{i} q_{j} & =q_{j} s_{i} & & \text { if }|i-j|>1 \\
s_{i} s_{j} q_{i} & =q_{j} s_{i} s_{j} & & \text { if }|i-j|=1 \\
q_{i} s_{i}=s_{i} q_{i} & =q_{i} & & \text { for all } i \\
q_{i} e_{j} & =e_{j} q_{i} & & \text { if } j \notin\{i, i+1\} \\
q_{i} e_{j} q_{i} & =q_{i} & & \text { if } j \in\{i, i+1\} \\
e_{j} q_{i} e_{j} & =e_{j} & & \text { if } j \in\{i, i+1\} .
\end{aligned}
$$

(The jump in labels from relation (R10) to (R18) will become clear shortly.) The next result was originally stated (in a different form) in [37, Theorem 1.11], and proved in [20, Theorem 36].

Theorem 2.6. The partition monoid, $\mathcal{P}_{n}$, has monoid presentation

$$
\langle S \cup E \cup Q:(\mathrm{R} 18-\mathrm{R} 33)\rangle
$$

via $\psi$.

\section{The singular rook partition monoid $\mathcal{R} \mathcal{P}_{n} \backslash \mathcal{S}_{n}$}

In this section, we obtain a (semigroup) presentation for $\mathcal{R} \mathcal{P}_{n} \backslash \mathcal{S}_{n}$, the singular part of the rook partition monoid $\mathcal{R} \mathcal{P}_{n}$; see Theorem 3.14. This presentation extends the presentation of $\mathcal{P}_{n} \backslash \mathcal{S}_{n}$ stated in Theorem [2.5, and uses the minimum number of generators (Theorem 3.15). We note that the method used in this section could also be used to derive a presentation for $\mathcal{R} \mathcal{P}_{n}$ itself (see Theorem 4.7) from that of $\mathcal{P}_{n}$ (Theorem 2.6). However, rather than duplicating the method for $\mathcal{R} \mathcal{P}_{n}$, we instead use Theorem 3.14 as a stepping stone towards proving Theorem 4.7 in Section 4 , leading to a shorter proof. It seems that this is the first time this method (of first finding a presentation for the singular subsemigroup) has been used, though we note some similarities to the author's recent work on (singular) symmetric inverse semigroups [22]. 
Now define a new alphabet $O=\left\{o_{1}, \ldots, o_{n}\right\}$, and for each $i$, let

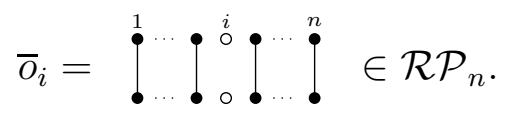

Note that if $A=\left\{i_{1}, \ldots, i_{k}\right\} \subseteq[n]$, then $\bar{o}_{A}$ (as defined in the previous section) may be factorised as $\bar{o}_{A}=\bar{o}_{i_{1}} \cdots \bar{o}_{i_{k}}$; in particular, $\bar{o}_{i}=\bar{o}_{\{i\}}$ for any $i \in[n]$. With this observation, the next result follows immediately from Proposition 2.3 and Theorem 2.5 ,

Proposition 3.1. The singular part of the rook partition monoid, $\mathcal{R} \mathcal{P}_{n} \backslash \mathcal{S}_{n}$, is generated (as a semigroup) by the set $\left\{\bar{e}_{1}, \ldots, \bar{e}_{n}\right\} \cup\left\{\bar{t}_{i j}: 1 \leq i<j \leq n\right\} \cup\left\{\bar{o}_{1}, \ldots, \bar{o}_{n}\right\}$.

Remark 3.2. The previous result shows that $\mathcal{R} \mathcal{P}_{n} \backslash \mathcal{S}_{n}$ is generated by its idempotents; this property is shared by many [7,21,24,56], but not all [13, 14, 17], diagram monoids. Under the above-mentioned embedding $\mathcal{R} \mathcal{P}_{n} \rightarrow \mathcal{P}_{n+1}$, the generators $\bar{o}_{i} \in \mathcal{R} \mathcal{P}_{n}$ are mapped to $\bar{t}_{i, n+1} \in \mathcal{P}_{n+1}$. See also [39].

By Proposition 3.1, we may define an epimorphism

$$
\Phi:(E \cup T \cup O)^{+} \rightarrow \mathcal{R} \mathcal{P}_{n} \backslash \mathcal{S}_{n}
$$

by $x \Phi=\bar{x}$ for each $x \in E \cup T \cup O$. Now consider the relations

$$
\begin{aligned}
o_{i}^{2} & =o_{i} & & \text { for all } i \\
o_{i} o_{j} & =o_{j} o_{i} & & \text { for distinct } i, j \\
o_{i} e_{j} & =e_{j} o_{i} & & \text { for distinct } i, j \\
o_{i} e_{i} O_{i} & =o_{i} & & \text { for all } i \\
e_{i} o_{i} e_{i} & =e_{i} & & \text { for all } i \\
t_{i j} o_{k} & =o_{k} t_{i j} & & \text { for any } i, j, k \\
t_{i j} o_{i}=t_{i j} o_{j} & =o_{i} o_{j} & & \text { for all } i, j .
\end{aligned}
$$

Our aim in this section is to show that $\mathcal{R} \mathcal{P}_{n} \backslash \mathcal{S}_{n}$ has (semigroup) presentation $\langle E \cup T \cup O:(\mathrm{R} 1-\mathrm{R} 17)\rangle$ via $\Phi$.

Since we already know $\Phi$ is surjective, it remains to show that ker $\Phi$ is generated by the relations (R1-R17). With this in mind, let $\sim$ be the congruence on $(E \cup T \cup O)^{+}$generated by relations $(\mathrm{R} 1-\mathrm{R} 17)$. For $w \in(E \cup T \cup O)^{+}$, write $\bar{w}=w \Phi \in \mathcal{R} \mathcal{P}_{n} \backslash \mathcal{S}_{n}$. For convenience, we will also write $\overline{1}=1$ and $1 \sim 1$ (even though the empty word 1 does not belong to $(E \cup T \cup O)^{+}$).

Lemma 3.3. We have $\sim \subseteq \operatorname{ker} \Phi$.

Proof. We need to show that each of relations (R1-R17) hold as equations in $\mathcal{R} \mathcal{P}_{n} \backslash \mathcal{S}_{n}$ when the words are replaced by their images under $\Phi$. We already know from Theorem 2.5 that this is the case for (R1-R10). The remaining relations may easily be checked diagramatically; we do this for (R17) in Figure 5, and leave the rest for the reader.

Establishing the reverse inclusion, $\operatorname{ker} \Phi \subseteq \sim$, forms the bulk of this section. The main step in doing this is to obtain a "word version" of Proposition 2.3, see Proposition 3.13, Our first aim is to show that any word over $(E \cup T \cup O)^{+}$is $\sim$-equivalent to an element of $O^{*}(E \cup T)^{*} O^{*}$; see Lemma 3.5, the proof of which requires the next technical result.

Lemma 3.4. Let $w \in(E \cup T)^{*}$ and $i \in[n]$. Then $w_{i} e_{i} \sim w_{1} w_{2} w_{3}$ for some $w_{1}, w_{3} \in O^{*}$ and $w_{2} \in(E \cup T)^{*}$. 

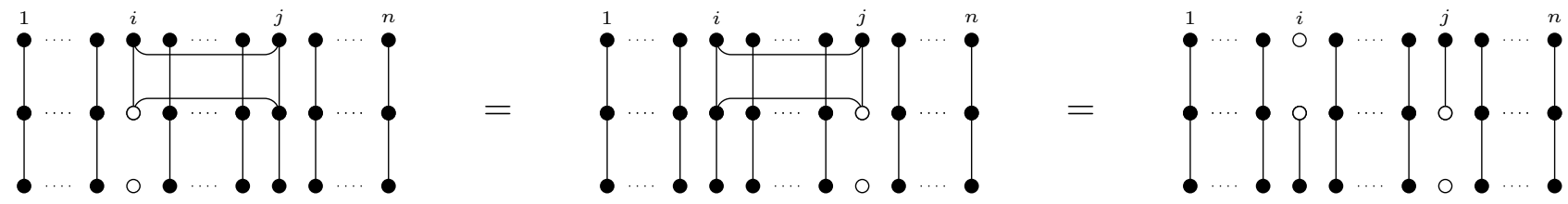

Figure 5: Diagrammatic proof of relation (R17): $\bar{t}_{i j} \bar{o}_{i}=\bar{t}_{i j} \bar{o}_{j}=\bar{o}_{i} \bar{o}_{j}$.

Proof. We prove the result by induction on $\ell(w)$, the length of $w$. If $\ell(w)=0$, then we are done (with $w_{1}=o_{i}, w_{2}=e_{i}$ and $w_{3}=1$ ), so suppose $\ell(w) \geq 1$, and write $w=u x$, where $x \in E \cup T$ (so $u \in(E \cup T)^{*}$ and $\left.\ell(u)=\ell(w)-1\right)$. We now consider separate cases, according to whether $x$ belongs to $E$ or $T$.

Case 1. First suppose $x=e_{j} \in E$. If $j=i$, then $w o_{i} e_{i}=u e_{i} o_{i} e_{i} \sim u e_{i}=w$, by (R15), and we are done (with $w_{1}=w_{3}=1$ and $w_{2}=w$ ). If $j \neq i$, then $w o_{i} e_{i}=u e_{j} o_{i} e_{i} \sim u o_{i} e_{i} e_{j}$, by (R2) and (R13), and we are done after applying an induction hypothesis to $u o_{i} e_{i}$.

Case 2. Next suppose $x=t_{j k} \in T$. If $i \notin\{j, k\}$, then $w o_{i} e_{i}=u t_{j k} o_{i} e_{i} \sim u o_{i} e_{i} t_{j k}$, by (R6) and (R16), and again we are done after applying an induction hypothesis. If $i \in\{j, k\}$, then, writing $\{l\}=\{j, k\} \backslash\{i\}$, we have $w_{i} e_{i}=u t_{j k} o_{i} e_{i} \sim u t_{j k} o_{l} e_{i} \sim u t_{j k} e_{i} o_{l}=w e_{i} o_{l}$, by (R17) and (R13), and we are done (with $w_{1}=1, w_{2}=w e_{i}$ and $w_{3}=o_{l}$ ).

Lemma 3.5. Let $w \in(E \cup T \cup O)^{+}$. Then $w \sim w_{1} w_{2} w_{3}$ for some $w_{1}, w_{3} \in O^{*}$ and $w_{2} \in(E \cup T)^{*}$.

Proof. This is clearly true if $\ell(w)=1$, so suppose $\ell(w) \geq 2$ and write $w=u x$, where $x \in E \cup T \cup O$. By an induction hypothesis, $u \sim u_{1} u_{2} u_{3}$ for some $u_{1}, u_{3} \in O^{*}$ and $u_{2} \in(E \cup T)^{*}$.

Case 1. If $x \in O$, then we are done, with $w_{1}=u_{1}, w_{2}=u_{2}$ and $w_{3}=u_{3} x$.

Case 2. If $x \in T$, then relation (R16) gives $u_{3} x \sim x u_{3}$, and we are done, with $w_{1}=u_{1}, w_{2}=u_{2} x$ and $w_{3}=u_{3}$.

Case 3. Finally, suppose $x=e_{i} \in E$. If $\ell\left(u_{3}\right)=0$, then we are done, so suppose $\ell\left(u_{3}\right) \geq 1$, and write $u_{3}=o_{j_{1}} \cdots o_{j_{k}}$; by (R11) and (R12), we may assume that $j_{1}<\cdots<j_{k}$. If $i \notin\left\{j_{1}, \ldots, j_{k}\right\}$, then $u_{3} e_{i} \sim e_{i} u_{3}$, by (R13), and we are done. So suppose $i \in\left\{j_{1}, \ldots, j_{k}\right\}$; say, $i=j_{l}$. Then $u_{3} e_{i} \sim o_{i} e_{i} v$, by (R12) and (R13), where $v=o_{j_{1}} \cdots o_{j_{l-1}} o_{j_{l+1}} \cdots o_{j_{k}}$. By Lemma 3.4, $u_{2} o_{i} e_{i} \sim v_{1} v_{2} v_{3}$ for some $v_{1}, v_{3} \in O^{*}$ and $v_{2} \in(E \cup T)^{*}$. Putting all this together, we have $w \sim u_{1} v_{1} v_{2} v_{3} v$, and we are done, with $w_{1}=u_{1} v_{1}, w_{2}=v_{2}$ and $w_{3}=v_{3} v$.

Next we wish to show (in Proposition 3.13) that the words $w_{1}, w_{2}, w_{3}$ from Lemma 3.5 may be chosen so that $\bar{w}_{1}, \bar{w}_{2}, \bar{w}_{3}$ correspond to the factorisation of $\bar{w} \in \mathcal{R} \mathcal{P}_{n} \backslash \mathcal{S}_{n}$ as in Proposition 2.3. To achieve this goal, we need several intermediate results.

For $A=\left\{i_{1}, \ldots, i_{k}\right\} \subseteq[n]$ with $i_{1}<\cdots<i_{k}$, we define the words

$$
o_{A}=o_{i_{1}} \cdots o_{i_{k}} \in O^{*} \quad \text { and } \quad t_{A}=t_{i_{1} i_{2}} t_{i_{2} i_{3}} \cdots t_{i_{k-1} i_{k}} \in T^{*} .
$$

Note that $o_{A}=1$ if $A=\emptyset$, while $t_{A}=1$ if $|A| \leq 1$. Note also that $o_{A} \Phi=\bar{o}_{A}$, agreeing with our earlier use of this notation.

Lemma 3.6. Let $A \subseteq[n]$ and let $i \in A$. Then $o_{A} \sim o_{i} t_{A} \sim t_{A} o_{i}$.

Proof. By (R16), it suffices to show that $o_{A} \sim o_{i} t_{A}$, and we do this by induction on $|A|$. If $|A|=1$, then there is nothing to show, since then $o_{A}=o_{i}$ and $t_{A}=1$. So suppose $|A| \geq 2$, and write 
$A=\left\{j_{1}, \ldots, j_{k}\right\}$ with $j_{1}<\cdots<j_{k}$. Then $i=j_{l}$ for some $1 \leq l \leq k$, and we write $B=A \backslash\{i\}$. Since $k=|A| \geq 2$, we must have $l>1$ or $l<k$ (or both). We will assume that $l<k$ (the other case is similar). Then

$$
\begin{aligned}
o_{A} & \sim o_{i} o_{B} & & \text { by (R12) } \\
& \sim o_{i} o_{j_{l+1}} t_{B} & & \text { by an induction hypothesis } \\
& \sim o_{i} t_{i j_{l+1}}\left(t_{j_{1} j_{2}} \cdots t_{j_{l-2} j_{l-1}} t_{j_{l-1} j_{l+1}} t_{j_{l+1} j_{l+2}} \cdots t_{j_{k-1} j_{k}}\right) & & \text { by (R16) and (R17) } \\
& \sim o_{i}\left(t_{j_{1} j_{2}} \cdots t_{j_{l-2} j_{l-1}} t_{i j_{l+1}} t_{j_{l-1} j_{l+1}} t_{j_{l+1} j_{l+2}} \cdots t_{j_{k-1} j_{k}}\right) & & \text { by (R4) } \\
& =o_{i}\left(t_{j_{1} j_{2}} \cdots t_{j_{l-2} j_{l-1}} t_{j_{l} j_{l+1}} t_{j_{l+1} j_{l-1}} t_{j_{l+1} j_{l+2}} \cdots t_{j_{k-1} j_{k}}\right) & & \\
& \sim o_{i}\left(t_{j_{1} j_{2}} \cdots t_{j_{l-2} j_{l-1}} t_{j_{l-1} j_{l}} t_{j_{l} j_{l+1}} t_{j_{l+1} j_{l+2}} \cdots t_{j_{k-1} j_{k}}\right) & & \text { by (R5) } \\
& =o_{i} t_{A} . & &
\end{aligned}
$$

Using the previous result, we may now strengthen Lemma 3.5 .

Corollary 3.7. Let $w \in(E \cup T \cup O)^{+}$. Then $w \sim w_{1} w_{2} w_{3}$ for some $w_{1}, w_{3} \in O^{*}$ and $w_{2} \in(E \cup T)^{*}$, with $\ell\left(w_{1}\right), \ell\left(w_{3}\right) \leq 1$.

Proof. By Lemma 3.5, $w \sim u_{1} u_{2} u_{3}$ for some $u_{1}, u_{3} \in O^{*}$ and $u_{2} \in(E \cup T)^{*}$. Then $u_{1} \sim o_{A}$ and $u_{3} \sim o_{B}$ for some (possibly empty) subsets $A, B \subseteq[n]$, by (R11) and (R12). If $A \neq \emptyset$, then choose some $i \in A$; if $B \neq \emptyset$, then choose some $j \in B$. Then

$$
w \sim u_{1} u_{2} u_{3} \sim o_{A} u_{2} o_{B} \sim \begin{cases}u_{2} & \text { if } A=\emptyset=B \\ o_{i}\left(t_{A} u_{2}\right) & \text { if } A \neq \emptyset=B \\ \left(u_{2} t_{B}\right) o_{j} & \text { if } A=\emptyset \neq B \\ o_{i}\left(t_{A} u_{2} t_{B}\right) o_{j} & \text { if } A \neq \emptyset \neq B\end{cases}
$$

by Lemma 3.6, and the proof is complete.

Next we wish to show how the relations may be used to move a generator from $O$ through a word from $(E \cup T)^{*}$ in certain circumstances. To do this, we require a result from [22] on (singular) symmetric inverse semigroups.

For distinct $i, j \in[n]$, we define the word $e_{i j}=e_{i} t_{i j} e_{j} \in(E \cup T)^{+}$, recalling that we are using symmetric notation for $t_{i j}=t_{j i}$. One easily checks that

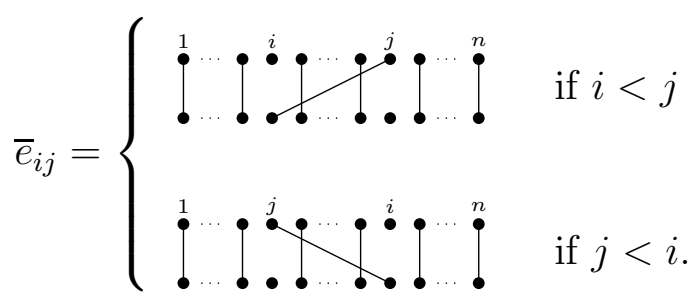

In particular, $\bar{e}_{i j} \neq \bar{e}_{j i}$. The next result is [22, Proposition 2.2].

Proposition 3.8. The singular part of the symmetric inverse monoid, $\mathcal{I}_{n} \backslash \mathcal{S}_{n}$, is generated (as a semigroup) by the set $\left\{\bar{e}_{i j}: i, j \in[n], i \neq j\right\}$.

Remark 3.9. Defining relations were also given in [22, Theorem 2.1] but we do not need those here. 
Lemma 3.10. Let $i, j \in[n]$ with $i \neq j$, and let $k \in\{i\}^{c}$. Then

$$
o_{k} e_{i j} \sim \begin{cases}e_{i j} o_{k} & \text { if } k \neq j \\ e_{i j} o_{i} & \text { if } k=j\end{cases}
$$

Proof. The case in which $k \neq j$ follows immediately from (R13) and (R16). We also have

$$
o_{j} e_{i j}=o_{j} e_{i} t_{i j} e_{j} \sim e_{i} o_{j} t_{i j} e_{j} \sim e_{i} t_{i j} o_{j} e_{j} \sim e_{i} t_{i j} o_{i} e_{j} \sim e_{i} t_{i j} e_{j} o_{i}=e_{i j} o_{i},
$$

by (R13), (R16), (R17), (R13), respectively.

As usual, for $\alpha \in \mathcal{I}_{n}$ and $i \in \operatorname{dom}(\alpha)$, we write $i \alpha$ for the unique element of $\operatorname{codom}(\alpha)$ such that $\left\{i,(i \alpha)^{\prime}\right\}$ is a block of $\alpha$. Note that Lemma 3.10 says that $o_{k} e_{i j} \sim e_{i j} o_{k \bar{e}_{i j}}$ for all $k \in\{i\}^{c}=\operatorname{dom}\left(\bar{e}_{i j}\right)$; compare with the above illustration(s) of $\bar{e}_{i j}$.

Corollary 3.11. Let $w \in(E \cup T)^{*}$ be such that $\bar{w} \in \mathcal{I}_{n}$. Then $o_{i} w \sim w o_{i \bar{w}}$ for any $i \in \operatorname{dom}(\bar{w})$.

Proof. The result is trivial if $\ell(w)=0$, so suppose $\ell(w) \geq 1$. In particular, $\bar{w} \in \mathcal{I}_{n} \backslash \mathcal{S}_{n}$, so $\bar{w}=$ $\bar{e}_{i_{1} j_{1}} \cdots \bar{e}_{i_{k} j_{k}}$ for some $i_{t}, j_{t}$, by Proposition 3.8. It follows from Theorem 2.5 that $w \sim e_{i_{1} j_{1}} \cdots e_{i_{k} j_{k}}$, and the result now follows from Lemma 3.10 and a simple induction on $k$.

For the proof of the next result, note that if

$$
\alpha=\left(\begin{array}{c|c|c}
A_{1} & \cdots & A_{r} \\
A_{1} & \cdots & A_{r}
\end{array}\right) \in \mathcal{J}_{n}
$$

then $\alpha=\overline{t_{A_{1}} \cdots t_{A_{r}}}$, where the words $t_{A}$ were defined before Lemma 3.6 .

Lemma 3.12. Suppose $i, j \in[n]$ and $w \in(E \cup T)^{*}$ are such that $i$ and $j^{\prime}$ belong to the same block $A \cup B^{\prime}$ of $\bar{w}$. Then $o_{i} w \sim w o_{j} \sim o_{i} u o_{j}$ for some $u \in(E \cup T)^{+}$such that $\bar{u}=\left(\bar{w} \backslash\left\{A \cup B^{\prime}\right\}\right) \cup\left\{A, B^{\prime}\right\}$.

Proof. Suppose first that $\ell(w)=0$. It follows that $w=1$ and $i=j$, in which case $o_{i} w=w o_{j}=$ $o_{i} \sim o_{i} e_{i} o_{i}$, by (R14), and we are done (with $u=e_{i}$ ). For the remainder of the proof, we assume that $\ell(w) \geq 1$. In particular, $\bar{w} \in \mathcal{P}_{n} \backslash \mathcal{S}_{n}$. Write

$$
\bar{w}=\left(\begin{array}{c|c|c|c|c|c}
A_{1} & \cdots & A_{r} & C_{1} & \cdots & C_{p} \\
\cline { 2 - 5 } & \ldots & B_{r} & D_{1} & \cdots & D_{q}
\end{array}\right),
$$

and let $\beta, \gamma, \delta$ be such that $\bar{w}=\beta \gamma \delta$, as in Proposition 2.1. Without loss of generality, we may suppose that $A=A_{r}, B=B_{r}$, and that $a_{r}=i$ and $b_{r}=j$. Let $w_{1}, w_{3} \in T^{*}$ and $w_{2} \in(E \cup T)^{+}$ be such that $\bar{w}_{1}=\beta, \bar{w}_{2}=\gamma$ and $\bar{w}_{3}=\delta$. Note that $w_{2} \neq 1$ since $r=\operatorname{rank}(\bar{w})<n$, and that $i \bar{w}_{2}=i \gamma=j$. Then

$$
\begin{aligned}
o_{i} w & \sim o_{i} w_{1} w_{2} w_{3} \\
& \sim w_{1} o_{i} w_{2} w_{3} \\
& \sim w_{1} w_{2} o_{j} w_{3} \\
& \sim w_{1} w_{2} w_{3} o_{j} \\
& \sim w_{j}
\end{aligned}
$$

by Theorem 2.5

by (R16)

by Corollary 3.11

by (R16)

by Theorem 2.5 . 
We also have

$$
\begin{aligned}
o_{i} w & \sim w_{1} o_{i} w_{2} w_{3} \\
& \sim w_{1} o_{i} e_{i} o_{i} w_{2} w_{3} \\
& \sim o_{i} w_{1} e_{i} w_{2} w_{3} o_{j} \\
& =o_{i} u o_{j},
\end{aligned}
$$

by Theorem 2.5 and (R16), as above

by Corollary 3.11 and (R16), as above

where $u=w_{1} e_{i} w_{2} w_{3}$. Note that

$$
\overline{e_{i} w_{2}}=\left(\begin{array}{c|c|c}
a_{1} & \ldots & a_{r-1} \\
b_{1} & \ldots & b_{r-1}
\end{array}\right) \quad \text { so that } \quad \bar{u}=\overline{w_{1} e_{i} w_{2} w_{3}}=\left(\begin{array}{c|c|c|c|c|c|c}
A_{1} & \ldots & A_{r-1} & A & C_{1} & \cdots & C_{p} \\
\cline { 2 - 2 } B_{1} & \ldots & B_{r-1} & B & D_{1} & \cdots & D_{q}
\end{array}\right) \text {, }
$$

completing the proof.

We are now ready to describe the promised normal forms for words over $E \cup T \cup O$. As in Proposition 2.3 , for the statement of the next result, we will allow ourselves to refer to the empty set $\emptyset$ as a block of any element of $\mathcal{P}_{n}$.

Proposition 3.13. Let $w \in(E \cup T \cup O)^{+}$. Then $w \sim o_{A} u o_{B}$ for some (possibly empty) $A, B \subseteq[n]$ and $u \in(E \cup T)^{+}$with $A$ and $B^{\prime}$ (possibly empty) blocks of $\bar{u}$.

Proof. By Corollary 3.7, $w \sim w_{1} v w_{2}$ for some $w_{1}, w_{2} \in O^{*}$ and $v \in(E \cup T)^{*}$, with $\ell\left(w_{1}\right), \ell\left(w_{2}\right) \leq 1$. If $\ell\left(w_{1}\right)=\ell\left(w_{2}\right)=0$, we are already done (with $A=B=\emptyset$ and $u=w$ ), so suppose this is not the case.

Case 1. Suppose first that $\ell\left(w_{1}\right)=1$ and $\ell\left(w_{2}\right)=0$, so $w \sim o_{i} v$ for some $i \in[n]$. Write $[i]_{\bar{v}}=A \cup B^{\prime}$, where $A, B \subseteq[n]$, noting that $A$ is non-empty (since $i \in A$ ), but $B$ may be empty. Since $A \cup B^{\prime}$ is a block of $\bar{v}$, it follows that $\bar{t}_{A} \bar{v}_{B}=\bar{v}$, so Theorem 2.5 gives $v \sim t_{A} v t_{B}$ (but note that this just says $1 \sim 1$ if $\ell(v)=0$ ).

Subcase 1.1. Suppose first that $B=\emptyset$. Then by Lemma 3.6 and the above calculations, we have $w \sim o_{i} v \sim o_{i} t_{A} v \sim o_{A} v$, so the proof is complete in this case (with $u=v$ ).

Subcase 1.2. Now suppose $B \neq \emptyset$, and choose some $j \in B$. By Lemma 3.12, $w \sim o_{i} v \sim o_{i} u o_{j}$ for some $u \in(E \cup T)^{+}$such that $\bar{u}=\left(\bar{v} \backslash\left\{A \cup B^{\prime}\right\}\right) \cup\left\{A, B^{\prime}\right\}$. Since $A$ and $B^{\prime}$ are blocks of $\bar{u}$, it again follows from Theorem 2.5 that $u \sim t_{A} u t_{B}$. Together with Lemma 3.6, we then obtain $w \sim o_{i} u o_{j} \sim o_{i} t_{A} u t_{B} o_{j} \sim o_{A} u o_{B}$, completing the proof in this case.

Case 2. The case in which $\ell\left(w_{1}\right)=0$ and $\ell\left(w_{2}\right)=1$ is similar to the previous case.

Case 3. Finally, suppose $\ell\left(w_{1}\right)=\ell\left(w_{2}\right)=1$, so $w \sim o_{i} v o_{j}$ for some $i, j \in[n]$. Write $[i]_{\bar{v}}=A \cup C^{\prime}$ and $\left[j^{\prime}\right]_{\bar{v}}=D \cup B^{\prime}$, noting that $A$ and $B$ are non-empty, but that $C$ and/or $D$ may be empty. (Note that it is possible that $[i]_{\bar{v}}=\left[j^{\prime}\right]_{\bar{v}}$, in which case $A=D$ and $B=C$, but this is included in the case that $C$ and $D$ are non-empty.)

Subcase 3.1. Suppose first that $D \neq \emptyset$, and let $k \in D$. Then $v o_{j} \sim o_{k} v$, by Lemma 3.12, so

$$
w \sim o_{i} v o_{j} \sim o_{i} o_{k} v \sim \begin{cases}o_{i} v & \text { by }(\mathrm{R} 11), \text { if } k=i \\ o_{i} t_{i k} v & \text { by }(\mathrm{R} 17) \text { and }(\mathrm{R} 16), \text { if } k \neq i .\end{cases}
$$

In either case, this reduces to Case 1.

Subcase 3.2. In similar fashion to the previous subcase, if $C \neq \emptyset$, then we may reduce to Case 2 .

Subcase 3.3. Finally, suppose $C=D=\emptyset$. Then $w \sim o_{i} v o_{j} \sim o_{i} t_{A} v t_{B} o_{j} \sim o_{A} v o_{B}$, by Theorem 2.5 and Lemma 3.6, and we are done, with $u=v$. 
We may now prove the main result of this section.

Theorem 3.14. The singular part of the rook partition monoid, $\mathcal{R} \mathcal{P}_{n} \backslash \mathcal{S}_{n}$, has semigroup presentation

$$
\langle E \cup T \cup O:(\mathrm{R} 1-\mathrm{R} 17)\rangle
$$

via $\Phi$.

Proof. It remains to show that $\operatorname{ker} \Phi \subseteq \sim$, so suppose $w_{1}, w_{2} \in(E \cup T \cup O)^{+}$are such that $\bar{w}_{1}=\bar{w}_{2}$. By Proposition [3.13, $w_{1} \sim o_{A} u_{1} o_{B}$ and $w_{2} \sim o_{C} u_{2} o_{D}$, for some (possibly empty) $A, B, C, D \subseteq[n]$ and $u_{1}, u_{2} \in(E \cup T)^{+}$such that $A$ and $B^{\prime}$ are (possibly empty) blocks of $\bar{u}_{1}$, and $C$ and $D^{\prime}$ are (possibly empty) blocks of $\bar{u}_{2}$. But then $\bar{o}_{A} \bar{u}_{1} \bar{o}_{B}=\bar{w}_{1}=\bar{w}_{2}=\bar{o}_{C} \bar{u}_{2} \bar{o}_{D}$. Proposition 2.3 then gives $A=C, B=D$ and $\bar{u}_{1}=\bar{u}_{2}$. Theorem 2.5 gives $u_{1} \sim u_{2}$. Putting this all together, we have $w_{1} \sim o_{A} u_{1} o_{B} \sim o_{A} u_{2} o_{B}=o_{C} u_{2} o_{D} \sim w_{2}$, completing the proof.

The next result shows that the generating set used in Theorem 3.14 has the smallest possible size among all generating sets for $\mathcal{R} \mathcal{P}_{n} \backslash \mathcal{S}_{n}$. Recall that the rank a semigroup $S$, denoted $\operatorname{rank}(S)$, is the smallest size of a generating set for $S$. If $S$ is generated by its idempotents, then the idempotent rank of $S$, denoted $\operatorname{idrank}(S)$, is the smallest size of a generating set consisting entirely of idempotents. For the proof of the next result, for $1 \leq i<j \leq n$, let $\varepsilon_{i j}$ be the equivalence relation on $[n]$ with $\{i, j\}$ as its only non-trivial equivalence class.

Theorem 3.15. We have $\operatorname{rank}\left(\mathcal{R} \mathcal{P}_{n} \backslash \mathcal{S}_{n}\right)=\operatorname{idrank}\left(\mathcal{R} \mathcal{P}_{n} \backslash \mathcal{S}_{n}\right)=\frac{n^{2}+3 n}{2}$

Proof. We know that $(E \cup T \cup O) \Phi$ is an idempotent generating set for $\mathcal{R} \mathcal{P}_{n} \backslash \mathcal{S}_{n}$. Since $|E \cup T \cup O|=$ $n+\left(\begin{array}{l}n \\ 2\end{array}\right)+n=\frac{n^{2}+3 n}{2}$, it follows that $\operatorname{idrank}\left(\mathcal{R} \mathcal{P}_{n} \backslash \mathcal{S}_{n}\right) \leq \frac{n^{2}+3 n}{2}$. Since also $\operatorname{rank}\left(\mathcal{R} \mathcal{P}_{n} \backslash \mathcal{S}_{n}\right) \leq$ $\operatorname{idrank}\left(\mathcal{R} \mathcal{P}_{n} \backslash \mathcal{S}_{n}\right)$, it remains to show that any generating set for $\mathcal{R} \mathcal{P}_{n} \backslash \mathcal{S}_{n}$ has size at least $\frac{n^{2}+3 n}{2}$. With this in mind, suppose $\mathcal{R} \mathcal{P}_{n} \backslash \mathcal{S}_{n}=\langle\Sigma\rangle$. We claim that:

(i) for all $i \in[n], \Sigma$ contains an element with domain $\{i\}^{c}$ and the block $\{i\}$;

(ii) for all $i \in[n], \Sigma$ contains an element with domain $\{i\}^{c}$ and the rook dot $i$; and

(iii) for all $1 \leq i<j \leq n, \Sigma$ contains an element with domain $[n]$ and kernel $\varepsilon_{i j}$.

In fact, the proofs of these are all very similar, so we just prove (i). With this in mind, let $i \in[n]$, and consider an expression $\bar{e}_{i}=\alpha_{1} \cdots \alpha_{k}$, where $\alpha_{1}, \ldots, \alpha_{k} \in \Sigma$. Then $n-1=\operatorname{rank}\left(\bar{e}_{i}\right)=$ $\operatorname{rank}\left(\alpha_{1} \cdots \alpha_{k}\right) \leq \operatorname{rank}\left(\alpha_{1}\right) \leq n-1$, so that $\operatorname{rank}\left(\alpha_{1}\right)=n-1$. We also have $\{i\}^{c}=\operatorname{dom}\left(\bar{e}_{i}\right)=$ $\operatorname{dom}\left(\alpha_{1} \cdots \alpha_{k}\right) \subseteq \operatorname{dom}\left(\alpha_{1}\right)$, so that $\operatorname{dom}\left(\alpha_{1}\right)$ is either $\{i\}^{c}$ or $[n]$. If the latter was the case, then (together with $\operatorname{rank}\left(\alpha_{1}\right)=n-1$ ) this would imply that $\operatorname{ker}\left(\alpha_{1}\right)=\varepsilon_{u v}$ for some $1 \leq u<v \leq n$, and this would give $\varepsilon_{u v}=\operatorname{ker}\left(\alpha_{1}\right) \subseteq \operatorname{ker}\left(\alpha_{1} \cdots \alpha_{k}\right)=\operatorname{ker}\left(\bar{e}_{i}\right)=\Delta$, a contradiction. So, in fact, $\operatorname{dom}\left(\alpha_{1}\right)=\{i\}^{c}$. It follows that either $\{i\}$ is a block of $\alpha_{1}$ or else $i$ is a rook dot of $\alpha_{1}$. If the latter was the case, then $i$ would also be a rook dot of $\alpha_{1} \cdots \alpha_{k}=\bar{e}_{i}$, a contradiction. So, in fact, $\{i\}$ is a block of $\alpha_{1}$. This completes the proof of (i). Finally, we note that $\Sigma$ has (at least) $n$ elements of type (i), $n$ of type (ii), and $\left(\begin{array}{l}n \\ 2\end{array}\right)$ of type (iii), so that $|\Sigma| \geq n+n+\left(\begin{array}{l}n \\ 2\end{array}\right)=\frac{n^{2}+3 n}{2}$.

Remark 3.16. Theorem 3.15 may also be proved using the general result [24, Theorem 5.10], utilising Green's relations and the regular *-semigroup structure of $\mathcal{R} \mathcal{P}_{n}$, which we have not investigated here. It would be interesting to study $\mathcal{R} \mathcal{P}_{n}$ with the machinery developed in [24, as it would allow one to study all the ideals of $\mathcal{R} \mathcal{P}_{n}$ (with $\mathcal{R} \mathcal{P}_{n} \backslash \mathcal{S}_{n}$ being the largest proper ideal). In particular, the following questions seem worthy of study: 
(i) What are the ranks of the other proper ideals of $\mathcal{R} \mathcal{P}_{n}$ ?

(ii) Are the other proper ideals of $\mathcal{R} \mathcal{P}_{n}$ generated by their idempotents? If so, what are their idempotent ranks? Are the ranks and idempotent ranks equal?

(iii) How many minimal-size (idempotent) generating sets are there for $\mathcal{R} \mathcal{P}_{n} \backslash \mathcal{S}_{n}$ ?

These questions are all answered for the proper ideals of partition monoid $\mathcal{P}_{n}$ in [24], as well as for the Brauer monoid and Jones monoid. See also [40].

\section{The rook partition monoid $\mathcal{R} \mathcal{P}_{n}$}

The goal of this section is to obtain a (monoid) presentation for the rook partition monoid $\mathcal{R} \mathcal{P}_{n}$; in fact, we obtain several such presentations (see Theorems 4.7, 4.11 and 4.12). Our approach makes crucial use of Theorem 3.14. Specifically, we first postulate a presentation for $\mathcal{R} \mathcal{P}_{n}$ (built up from the presentation of $\mathcal{P}_{n}$ in Theorem [2.6), and then show that the presentation for $\mathcal{R P}_{n} \backslash \mathcal{S}_{n}$ from Theorem 3.14 can be embedded (in a certain sense) in the stated presentation for $\mathcal{R P}_{n}$ (see Lemmas 4.4 and 4.6).

As with Proposition 3.1, the next result follows from Proposition 2.3 and Theorem 2.6 .

Proposition 4.1. The rook partition monoid, $\mathcal{R} \mathcal{P}_{n}$, is generated (as a monoid) by the set

$$
\left\{\bar{s}_{1}, \ldots, \bar{s}_{n-1}\right\} \cup\left\{\bar{e}_{1}, \ldots, \bar{e}_{n}\right\} \cup\left\{\bar{q}_{1}, \ldots, \bar{q}_{n-1}\right\} \cup\left\{\bar{o}_{1}, \ldots, \bar{o}_{n}\right\} .
$$

Consider the alphabets

$$
S=\left\{s_{1}, \ldots, s_{n-1}\right\}, \quad E=\left\{e_{1}, \ldots, e_{n}\right\}, \quad Q=\left\{q_{1}, \ldots, q_{n-1}\right\}, \quad O=\left\{o_{1}, \ldots, o_{n}\right\},
$$

as defined earlier. By Proposition 4.1, we may define a (monoid) epimorphism

$$
\Psi:(S \cup E \cup Q \cup O)^{*} \rightarrow \mathcal{R P}_{n}
$$

by $x \Psi=\bar{x}$ for each $x \in S \cup E \cup Q \cup O$. Consider also the relations

$$
\begin{aligned}
o_{i}^{2} & =o_{i} & & \text { for all } i \\
o_{i} o_{j} & =o_{j} o_{i} & & \text { for distinct } i, j \\
s_{i} O_{j} & =o_{j} s_{i} & & \text { if } j \notin\{i, i+1\} \\
s_{i} O_{i} & =o_{i+1} s_{i} & & \text { for all } i \\
o_{i} o_{i+1} s_{i} & =o_{i} O_{i+1} & & \text { for all } i \\
o_{i} e_{j} & =e_{j} o_{i} & & \text { for distinct } i, j \\
o_{i} e_{i} O_{i} & =o_{i} & & \text { for all } i \\
e_{i} o_{i} e_{i} & =e_{i} & & \text { for all } i \\
q_{i} O_{j} & =o_{j} q_{i} & & \text { for all } i, j \\
q_{i} o_{i}=q_{i} o_{i+1} & =o_{i} o_{i+1} & & \text { for all } i .
\end{aligned}
$$

Our goal in this section is to show that $\mathcal{R} \mathcal{P}_{n}$ has (monoid) presentation $\langle S \cup E \cup Q \cup O:(\mathrm{R} 18-\mathrm{R} 43)\rangle$ via $\Psi$.

Since we already know $\Psi$ is surjective, it remains to show that $\operatorname{ker} \Psi$ is generated by the relations (R18-R43). With this in mind, let $\approx$ denote the congruence on $(S \cup E \cup Q \cup O)^{*}$ generated by relations (R18-R43). Without causing confusion, we will write $\bar{w}=w \Psi$ for all $w \in(S \cup E \cup Q \cup O)^{*}$. As with Lemma 3.3, the next result may easily be proved diagrammatically. 
Lemma 4.2. We have $\approx \subseteq \operatorname{ker} \Psi$.

To establish the reverse containment, $\operatorname{ker} \Psi \subseteq \approx$, we need to show that $\bar{u}=\bar{v} \Rightarrow u \approx v$ for all $u, v \in(S \cup E \cup Q \cup O)^{*}$. The next result shows that this is the case for words $u, v$ in certain subsets of $(S \cup E \cup Q \cup O)^{*}$.

Proposition 4.3. If $u, v \in(S \cup E \cup Q)^{*}$ or $u, v \in(S \cup O)^{*}$, then $\bar{u}=\bar{v} \Rightarrow u \approx v$.

Proof. This is clearly the case for $u, v \in(S \cup E \cup Q)^{*}$, since (R18-R33) are defining relations for $\mathcal{P}_{n}=\langle(S \cup E \cup Q) \Psi\rangle$ (Theorem 2.6). Similarly, (R18-R20, R34-R38) constitute defining relations for $\mathcal{R}_{n}=\langle(S \cup O) \Psi\rangle$ (see [30, Theorem 4.8] and [19, Proposition 3.22]).

For a word $w=s_{i_{1}} \cdots s_{i_{k}} \in S^{*}$, we write $w^{-1}=s_{i_{k}} \cdots s_{i_{1}}$. Note that $w w^{-1} \approx w^{-1} w \approx 1$ for all $w \in S^{*}$, by (R18). For $1 \leq i<j \leq n$, define words $\sigma_{i j}=s_{i+1} \cdots s_{j-1}$ (which is empty if $j=i+1$ ) and $\tau_{i j}=\tau_{j i}=\sigma_{i j}^{-1} q_{i} \sigma_{i j}$. Note that $\bar{\tau}_{i j}=\bar{t}_{i j}$, as shown in Figure 6. Note also that $\tau_{i, i+1}=q_{i}$ for all $i$.

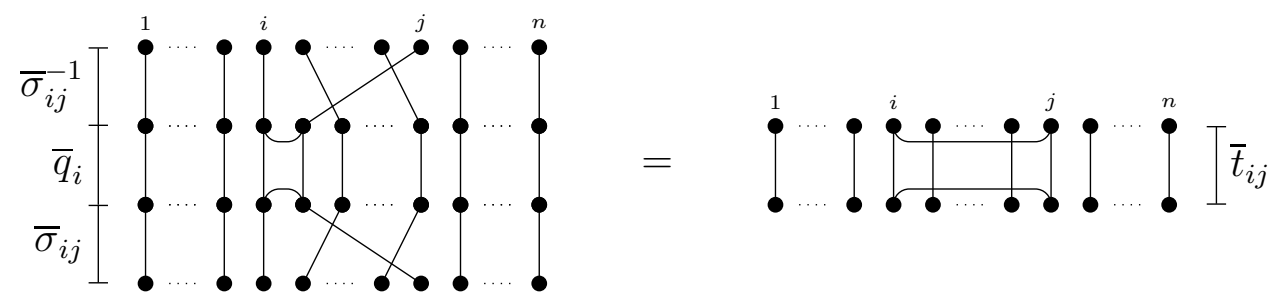

Figure 6: Diagrammatic proof that $\bar{\tau}_{i j}=\bar{t}_{i j}$.

As stated earlier, our strategy involves (somehow) linking the presentations $\langle E \cup T \cup O:(\mathrm{R} 1-\mathrm{R} 17)\rangle$ and $\langle S \cup E \cup Q \cup O:(\mathrm{R} 18-\mathrm{R} 43)\rangle$. In order to make this link explicit, we define a homomorphism

$$
\rho:(E \cup T \cup O)^{+} \rightarrow(S \cup E \cup Q \cup O)^{*}
$$

by $e_{i} \rho=e_{i}$ and $o_{i} \rho=o_{i}$ (for each $1 \leq i \leq n$ ) and $t_{i j} \rho=\tau_{i j}$ (for each $1 \leq i<j \leq n$ ). It follows that $\bar{w}=\overline{w \rho}$ (i.e., $w \Phi=(w \rho) \Psi)$ for all $w \in(E \cup T \cup O)^{+}$. Note that $\operatorname{im}(\rho)$ is the subsemigroup of $(S \cup E \cup Q \cup O)^{*}$ generated by $E \cup O \cup\left\{\tau_{i j}: 1 \leq i<j \leq n\right\}$.

Recall that $\sim$ is the congruence on $(E \cup T \cup O)^{+}$generated by relations $(\mathrm{R} 1-\mathrm{R} 17)$.

Lemma 4.4. For any $u, v \in(E \cup T \cup O)^{+}, u \sim v \Rightarrow u \rho \approx v \rho$.

Proof. First note that since $\sim$ is generated by relations $(\mathrm{R} 1-\mathrm{R} 17)$, it suffices to prove the result for each relation $u=v$ from $(\mathrm{R} 1-\mathrm{R} 17)$. Since $w \rho \in(S \cup E \cup Q)^{+}$for all $w \in(E \cup T)^{+}$, and since (as noted above) $\bar{w}=\overline{w \rho}$ for all $w \in(E \cup T)^{+}$, it follows from Proposition 4.3 that the result is true for relations (R1-R10). The result is also clearly true for relations (R11-R15) since these are precisely relations (R34), (R35) and (R39-41). It therefore remains to show that:

(i) $\tau_{i j} o_{k} \approx o_{k} \tau_{i j}$ for distinct $i, j$ and any $k$; and

(ii) $\tau_{i j} o_{i} \approx \tau_{i j} o_{j} \approx o_{i} o_{j}$ for distinct $i, j$.

Beginning with (i), let $i, j, k \in[n]$ with $i \neq j$. Put $l=k \bar{\sigma}_{i j}^{-1}$. Then $o_{l} \sigma_{i j} \approx \sigma_{i j} o_{k}$ and $\sigma_{i j}^{-1} o_{l} \approx o_{k} \sigma_{i j}^{-1}$ by Proposition 4.3 (and a simple diagrammatic check). Together with (R42), we obtain

$$
\tau_{i j} o_{k}=\sigma_{i j}^{-1} q_{i} \sigma_{i j} o_{k} \approx \sigma_{i j}^{-1} q_{i} o_{l} \sigma_{i j} \approx \sigma_{i j}^{-1} o_{l} q_{i} \sigma_{i j} \approx o_{k} \sigma_{i j}^{-1} q_{i} \sigma_{i j}=o_{k} \tau_{i j}
$$

For (ii), let $i, j \in[n]$ with $i \neq j$. Then, by Proposition 4.3, (R43) and (R18), we have

$$
\tau_{i j} o_{i}=\sigma_{i j}^{-1} q_{i} \sigma_{i j} o_{i} \approx \sigma_{i j}^{-1} q_{i} o_{i} \sigma_{i j} \approx \sigma_{i j}^{-1} o_{i} o_{i+1} \sigma_{i j} \approx o_{i} \sigma_{i j}^{-1} \sigma_{i j} o_{j} \approx o_{i} o_{j} .
$$

A similar calculation yields $\tau_{i j} o_{j} \approx o_{i} o_{j}$. As noted above, this completes the proof. 
Lemma 4.5. Let $1 \leq i \leq n-1$ and $x \in E \cup O \cup\left\{\tau_{i j}: 1 \leq i<j \leq n\right\}$. Then $s_{i} x$ and $x s_{i}$ are $\approx$-equivalent to an element of $\operatorname{im}(\rho)$.

Proof. We consider separate cases, depending on whether $x$ belongs to $E, O$ or $\left\{\tau_{i j}: 1 \leq i<j \leq n\right\}$.

Case 1. Suppose first that $x \in E$. One may check diagramatically that

$$
\begin{array}{rlrl}
\bar{s}_{i} \bar{e}_{j}=\bar{e}_{j} \bar{s}_{i} & =\bar{e}_{j} \bar{\tau}_{i+1, j} \bar{e}_{i+1} \bar{\tau}_{i, i+1} \bar{e}_{i} \bar{\tau}_{i j} \bar{e}_{j} & & \\
\bar{s}_{i} \bar{e}_{i}=\bar{e}_{i+1} \bar{s}_{i} & =\bar{e}_{i+1} \bar{\tau}_{i, i+1} \bar{e}_{i} & \\
\bar{s}_{i} \bar{e}_{i+1}=\bar{e}_{i} \bar{s}_{i} & =\bar{e}_{i} \bar{\tau}_{i, i+1} \bar{e}_{i+1} . &
\end{array}
$$

It follows by Proposition 4.3 that

$$
\begin{array}{rlr}
s_{i} e_{j} \approx e_{j} s_{i} & \approx e_{j} \tau_{i+1, j} e_{i+1} \tau_{i, i+1} e_{i} \tau_{i j} e_{j} & \\
s_{i} e_{i} \approx e_{i+1} s_{i} & \approx e_{i+1} \tau_{i, i+1} e_{i} & \\
s_{i} e_{i+1} \approx e_{i} s_{i} & \approx e_{i} \tau_{i, i+1} e_{i+1}, &
\end{array}
$$

completing the proof in this case.

Case 2. Next suppose $x=o_{j} \in O$, and put $k=j \bar{s}_{i}$ (so also $j=k \bar{s}_{i}$ ). Then $o_{j} s_{i} \approx s_{i} o_{k}$ and $s_{i} o_{j} \approx o_{k} s_{i}$, by Proposition 4.3. Together with (R40), we deduce that

$$
s_{i} o_{j} \approx s_{i} o_{j} e_{j} o_{j} \approx o_{k}\left(s_{i} e_{j}\right) o_{j} \quad \text { and } \quad o_{j} s_{i} \approx o_{j} e_{j} o_{j} s_{i} \approx o_{j}\left(e_{j} s_{i}\right) o_{k} .
$$

By the previous case, $s_{i} e_{j}$ and $e_{j} s_{i}$ are both $\approx$-equivalent to an element of $\operatorname{im}(\rho)$, so the proof is complete in this case also.

Case 3. Finally, suppose $x=\tau_{j k}$ for some $1 \leq j<k \leq n$, and put $u=j \bar{s}_{i}$ and $v=k \bar{s}_{i}$. By Proposition 4.3, we have $\tau_{j k} s_{i} \approx s_{i} \tau_{u v}$ and $\tau_{j k} \approx \tau_{j k} e_{j} \tau_{j k}$. It then follows that

$$
\tau_{j k} s_{i} \approx \tau_{j k} e_{j} \tau_{j k} s_{i} \approx \tau_{j k}\left(e_{j} s_{i}\right) \tau_{u v}
$$

and, again, it follows from Case 1 that $\tau_{j k} s_{i}$ is $\approx$-equivalent to an element of $\operatorname{im}(\rho)$. A similar calculation shows that this is the case also for $s_{i} \tau_{j k}$.

Lemma 4.6. Let $w \in(S \cup E \cup Q \cup O)^{*} \backslash S^{*}$. Then $w$ is $\approx$-equivalent to an element of $\operatorname{im}(\rho)$.

Proof. Put $\Sigma=E \cup O \cup\left\{\tau_{i j}: 1 \leq i<j \leq n\right\}$, noting that $\operatorname{im}(\rho)=\langle\Sigma\rangle$ is the subsemigroup of $(S \cup E \cup Q \cup O)^{*}$ generated by $\Sigma$. Since $Q \subseteq \Sigma\left(\right.$ as $\left.q_{i}=\tau_{i, i+1}\right)$, it suffices to show that every element of $\langle\Sigma \cup S\rangle \backslash S^{*}$ is $\approx$-equivalent to an element of $\operatorname{im}(\rho)$. With this in mind, let $w \in\langle\Sigma \cup S\rangle \backslash S^{*}$, and write $w=x_{1} \cdots x_{k}$, where $x_{1}, \ldots, x_{k} \in \Sigma \cup S$. Denote by $l$ the number of factors $x_{i}$ that belong to $S$. We proceed by induction on $l$. If $l=0$, then we already have $w \in\langle\Sigma\rangle=\operatorname{im}(\rho)$, so suppose $l \geq 1$. Since $w \notin S^{*}$, there exists $1 \leq i \leq k-1$ such that either (i) $x_{i} \in S$ and $x_{i+1} \in \Sigma$, or (ii) $x_{i} \in \Sigma$ and $x_{i+1} \in S$. In either case, Lemma 4.5 tells us that $x_{i} x_{i+1} \approx u$ for some $u \in \operatorname{im}(\rho)=\langle\Sigma\rangle$. But then $w \approx\left(x_{1} \cdots x_{i-1}\right) u\left(x_{i+2} \cdots x_{k}\right)$, and we are done, after applying an induction hypothesis (noting that $\left(x_{1} \cdots x_{i-1}\right) u\left(x_{i+2} \cdots x_{k}\right)$ has $l-1$ factors from $\left.S\right)$.

With these preliminary results in place, we may now prove the first of the main results of this section.

Theorem 4.7. The rook partition monoid, $\mathcal{R} \mathcal{P}_{n}$, has monoid presentation

$$
\langle S \cup E \cup Q \cup O:(\mathrm{R} 18-\mathrm{R} 43)\rangle
$$

via $\Psi$. 
Proof. It remains to show that $\operatorname{ker} \Psi \subseteq \approx$, so suppose $w_{1}, w_{2} \in(S \cup E \cup Q \cup O)^{*}$ are such that $\bar{w}_{1}=\bar{w}_{2}$. If $\bar{w}_{1} \in \mathcal{S}_{n}$, then $w_{1}, w_{2} \in S^{*}$, and $w_{1} \approx w_{2}$, using only relations (R18-R20). For the remainder of the proof, suppose $\bar{w}_{1} \notin \mathcal{S}_{n}$. It follows that $w_{1}, w_{2} \in(S \cup E \cup Q \cup O)^{*} \backslash S^{*}$. So, by Lemma 4.6, $w_{1} \approx u_{1} \rho$ and $w_{2} \approx u_{2} \rho$ for some $u_{1}, u_{2} \in(E \cup T \cup O)^{+}$. We then have

$$
\bar{u}_{1}=\overline{u_{1} \rho}=\bar{w}_{1}=\bar{w}_{2}=\overline{u_{2} \rho}=\bar{u}_{2},
$$

so that $u_{1} \sim u_{2}$, by Theorem 3.14, Lemma 4.4 then gives $u_{1} \rho \approx u_{2} \rho$, so that $w_{1} \approx w_{2}$.

\subsection{A presentation for $\mathcal{R} \mathcal{P}_{n}$ on $n+2$ generators}

The presentation from Theorem 4.7 uses $4 n-2$ generators. In this section, we use Tietze transformations to reduce the size of the generating set, thereby obtaining a presentation (Theorem 4.11) in terms of $S$ and three more generators. With this in mind, we rename $e=e_{1}, q=q_{1}, o=o_{1}$. Define words

$$
\begin{array}{cl}
c_{i}=s_{1} \cdots s_{i-1}, \quad E_{i}=c_{i}^{-1} e c_{i}, \quad O_{i}=c_{i}^{-1} o c_{i} & \text { for each } 1 \leq i \leq n \\
d_{j}=s_{2} \cdots s_{j} s_{1} \cdots s_{j-1}, \quad Q_{j}=d_{j}^{-1} q d_{j} & \text { for each } 1 \leq j \leq n-1 .
\end{array}
$$

Note that $c_{1}=d_{1}=1, E_{1}=e, O_{1}=o$ and $Q_{1}=q$. Diagramatically, one may check (in similar fashion to Figure 6) that

$$
\bar{e}_{i}=\bar{E}_{i}, \quad \bar{o}_{i}=\bar{O}_{i}, \quad \bar{q}_{j}=\bar{Q}_{j} \quad \text { for each } i, j .
$$

It follows from Theorem 4.7 that

$$
e_{i} \approx E_{i}, \quad o_{i} \approx O_{i}, \quad q_{j} \approx Q_{j} \quad \text { for each } i, j .
$$

So we may transform the above presentation into $\left\langle S \cup\{e, q, o\}:(\mathrm{R} 18-\mathrm{R} 43)^{\prime}\right\rangle$, where each relation $(\mathrm{R} k)^{\prime}$ is obtained from $(\mathrm{R} k)$ by replacing each letter $e_{i}, o_{i}, q_{j}$ by the words $E_{i}, O_{i}, Q_{j}$, respectively. This presentation is via the map $\xi:(S \cup\{e, q, o\})^{*} \rightarrow \mathcal{R} \mathcal{P}_{n}$ defined to be the restriction to $(S \cup\{e, q, o\})^{*}$ of $\Psi:(S \cup E \cup Q \cup O)^{*} \rightarrow \mathcal{R} \mathcal{P}_{n}$. Now consider the relations

$$
\begin{array}{rlrl}
s_{i}^{2} & =1 & & \text { for all } i \\
s_{i} s_{j} & =s_{j} s_{i} & & \text { if }|i-j|>1 \\
s_{i} s_{j} s_{i} & =s_{j} s_{i} s_{j} & & \text { if }|i-j|=1 \\
e^{2}=e & =e q e=e o e & & \\
q^{2}=q=q e q & =q s_{1}=s_{1} q & & \\
e s_{i} & =s_{i} e & & \\
q s_{i} & =s_{i} q & & \\
s_{1} e s_{1} e=e s_{1} & e s_{1}=e s_{1} e & & \\
q s_{2} q s_{2} & =s_{2} q s_{2} q & & \\
q\left(s_{2} s_{1} s_{3} s_{2}\right) q\left(s_{2} s_{1} s_{3} s_{2}\right) & =\left(s_{2} s_{1} s_{3} s_{2}\right) q\left(s_{2} s_{1} s_{3} s_{2}\right) q \\
q\left(s_{2} s_{1} e s_{1} s_{2}\right) & =\left(s_{2} s_{1} e s_{1} s_{2}\right) q & & \\
o^{2}=o & =o e o & & \text { if } i \geq 2 \\
o s_{i} & =s_{i} O & &
\end{array}
$$


It is easy to check, diagramatically, that each of relations (R44-R59) hold in $\mathcal{R} \mathcal{P}_{n}$ when the words are replaced by their images under $\xi$. So we may add them to the presentation, obtaining the presentation $\left\langle S \cup\{e, q, o\}:(\mathrm{R} 18-\mathrm{R} 43)^{\prime},(\mathrm{R} 44-\mathrm{R} 59)\right\rangle$. Our goal is to show that the relations (R18-R43)' may be removed; see Theorem 4.11. With this in mind, write $\approx$ for the congruence on $(S \cup\{e, q, o\})^{*}$ generated by $(\mathrm{R} 44-\mathrm{R} 59)$. For $w \in(S \cup\{e, q, o\})^{*}$, write $\bar{w}=w \xi$.

Proposition 4.8. If $u, v \in(S \cup\{e, q\})^{*}$ or $u, v \in(S \cup\{o\})^{*}$, then $\bar{u}=\bar{v} \Rightarrow u \approx v$.

Proof. The proof is similar to that of Proposition 4.3, since (R44-R54) include defining relations for $\mathcal{P}_{n}=\langle S \xi \cup\{\bar{e}, \bar{q}\}\rangle$ (see [20, Theorem 32]), while (R44-R45) and (R55-R57) include defining relations for $\mathcal{R}_{n}=\langle S \xi \cup\{\bar{o}\}\rangle$ (see [70] or [27]).

In particular, we may remove any of the relations $(\mathrm{R} k)^{\prime}$ involving only words over $S \cup\{e, q\}$ or only words over $S \cup\{o\}$. In this way, we may remove relations (R18-R38)'. The next two results follow immediately from Proposition 4.8 (and simple diagrammatic checks).

Corollary 4.9. Let $w \in S^{*}$ and $i \in[n]$. Then $w^{-1} E_{i} w \approx E_{i \bar{w}}$ and $w^{-1} O_{i} w \approx O_{i \bar{w}}$.

Corollary 4.10. Let $w \in S^{*}$ and $1 \leq i \leq n-1$, with $(i+1) \bar{w}=i \bar{w}+1$. Then $w^{-1} Q_{i} w \approx Q_{i \bar{w}}$.

Theorem 4.11. The rook partition monoid, $\mathcal{R} \mathcal{P}_{n}$, has monoid presentation

$$
\langle S \cup\{e, q, o\}:(\mathrm{R} 44-\mathrm{R} 59)\rangle
$$

via $\xi$.

Proof. So far, we have transformed the presentation into $\left\langle S \cup\{e, q, o\}:(\mathrm{R} 39-\mathrm{R} 43)^{\prime}\right.$, (R44-R59) . It remains to show that relations (R39-R43)' may be removed.

$(\mathbf{R 3 9})^{\prime}$. We need to show that $O_{i} E_{j} \approx E_{j} O_{i}$ if $i \neq j$. Let $w \in S^{*}$ be such that $1 \bar{w}=j$ and $2 \bar{w}=i$. Then

$$
O_{i} E_{j} \approx w^{-1} O_{2} w w^{-1} E_{1} w \approx w^{-1} O_{2} E_{1} w \approx w^{-1} E_{1} O_{2} w \approx w^{-1} E_{1} w w^{-1} O_{2} w \approx E_{j} O_{i},
$$

by Corollary 4.9, (R44) and (R58), the latter of which says " $E_{1} O_{2}=O_{2} E_{1}$ ".

$(\mathbf{R} 40)^{\prime}$ and $(\mathbf{R} 41)^{\prime}$. Here we have $O_{i} E_{i} O_{i}=c_{i}^{-1} o c_{i} c_{i}^{-1} e c_{i} c_{i}^{-1} o c_{i} \approx c_{i}^{-1} o e o c_{i} \approx c_{i}^{-1} o c_{i}=O_{i}$, by $(\mathrm{R} 44)$ and (R55). An almost identical calculation (using (R47) instead of (R55)) deals with relation (R41)'.

$(\mathbf{R} 42)^{\prime}$. We must show that $Q_{i} O_{j} \approx O_{j} Q_{i}$ for any $i, j$. Suppose first that $j \notin\{i, i+1\}$. Choose $w \in S^{*}$ such that $1 \bar{w}=i, 2 \bar{w}=i+1$ and $3 \bar{w}=j$. Then

$$
Q_{i} O_{j} \approx w^{-1} Q_{1} w w^{-1} O_{3} w \approx w^{-1} Q_{1} O_{3} w \approx w^{-1} O_{3} Q_{1} w \approx w^{-1} O_{3} w w^{-1} Q_{1} w \approx O_{j} Q_{i},
$$

by Corollaries 4.9 and 4.10, and relations (R44) and (R59), the latter of which says " $Q_{1} O_{3}=O_{3} Q_{1}$ ". Next, note that for any $u \in S^{*}$ with $1 \bar{u}=i$ and $2 \bar{u}=i+1, Q_{i} O_{i} \approx u^{-1}$ qou $\approx u^{-1}$ oqu $\approx O_{i} Q_{i}$, by Corollaries 4.9 and 4.10, and relations (R44) and (R57). A similar calculation gives $Q_{i} O_{i+1} \approx O_{i+1} Q_{i}$.

$(\mathbf{R} 43)^{\prime}$. Again, let $u \in S^{*}$ be such that $1 \bar{u}=i$ and $2 \bar{u}=i+1$. Then $Q_{i} O_{i} \approx u^{-1} Q_{1} O_{1} u \approx$ $u^{-1} O_{1} O_{2} u \approx O_{i} O_{i+1}$, by Corollaries 4.9 and 4.10, and relations (R44) and (R57), the relevant part of the latter of which says " $O_{1} Q_{1}=O_{1} O_{2}$ ". Finally, using Proposition 4.8 and the previous calculation, we also have $Q_{i} O_{i+1}=Q_{i} s_{i} O_{i} s_{i} \approx Q_{i} O_{i} s_{i} \approx O_{i} O_{i+1} s_{i} \approx O_{i} O_{i+1}$. This completes the proof. 


\subsection{A presentation for $\mathcal{R} \mathcal{P}_{n}$ on 5 generators}

We continue to reduce the presentation $\langle S \cup\{e, q, o\}:(\mathrm{R} 44-\mathrm{R} 59)\rangle$ from Theorem 4.11 in order to obtain a presentation with the minimal number of generators, making use of a 2-generator presentation for $\mathcal{S}_{n}$ [65]. In fact, the generating set $S \cup\{e, q, o\}$ is already of minimal size when $n=2$ (see Theorem 4.14 and Remark 4.15), so we will assume $n \geq 3$ for the rest of this section.

We now rename $s=s_{1}$, and add the new generator $c$, along with the relation $c=s_{1} \cdots s_{n-1}$. It is easy to check, diagrammatically, that $\bar{s}_{i+1}=\bar{c}^{i} \bar{s} \bar{c}^{-i}=\bar{c}^{i} \bar{s} \bar{c}^{n-i}$ for all $2 \leq i \leq n-2$; here we write $\bar{c}=\bar{s}_{1} \cdots \bar{s}_{n-1}$. It follows that we may remove the generators $s_{2}, \ldots, s_{n-1}$ from the presentation, and replace their every occurrence in the relations by the words $S_{i+1}=c^{i} s c^{n-i}$ (for each $1 \leq i \leq n-2$ ). (We also define $S_{1}=s$.) The result of doing this to relation $(\mathrm{R} k)$ will be denoted $(\mathrm{R} k)^{\prime}$. So we have transformed the presentation to

$$
\left\langle s, c, e, q, o:(\mathrm{R} 44-\mathrm{R} 59)^{\prime}, c=S_{1} \cdots S_{n-1}\right\rangle .
$$

This presentation is via the map

$$
\zeta:\{s, c, e, q, o\}^{*} \rightarrow \mathcal{R} \mathcal{P}_{n}: x \mapsto \bar{x} .
$$

Now consider the relations

$$
\begin{gathered}
c^{n}=(s c)^{n-1}=s^{2}=\left(c^{i} s c^{n-i} s\right)^{2}=1 \quad \text { for all } 2 \leq i \leq \frac{n}{2} \\
e^{2}=e=e q e=e o e=s c e c^{n-1} s=\csc ^{n-1} e c s c^{n-1} \\
q^{2}=q=q e q=q s=s q=c^{2} s c^{n-2} q c^{2} s c^{n-2}=c^{n-1} \operatorname{scsc}^{n-1} q c s c^{n-1} s c \\
\text { sese }=e s e s=e s e \\
q c q c^{n-1}=c q c^{n-1} q \\
q c^{2} q c^{n-2}=c^{2} q c^{n-2} q \\
q c^{2} e c^{n-2}=c^{2} e c^{n-2} q \\
o^{2}=o=o e o=s c o c^{n-1} s=c s c^{n-1} o s c^{n-1} \\
\text { soso }=o s o s=o s o=o q=q o \\
q c^{2} \text { os } c^{n-2}=c^{2} o c^{n-2} q \\
e s o s=s o s e .
\end{gathered}
$$

Theorem 4.12. The rook partition monoid, $\mathcal{R} \mathcal{P}_{n}$, has monoid presentation

$$
\langle s, c, e, q, o:(\mathrm{R} 60-\mathrm{R} 70)\rangle
$$

via $\zeta$.

Proof. Write $\diamond$ for the congruence on $\{s, c, e, q, o\}^{*}$ generated by relations (R60-R70). We already know that $\zeta$ is surjective, and it is easy to check that $\diamond \subseteq \operatorname{ker} \zeta$, so we may add relations (R60-R70) to obtain the presentation

$$
\left\langle s, c, e, q, o:(\mathrm{R} 60-\mathrm{R} 70),(\mathrm{R} 44-\mathrm{R} 59)^{\prime}, c=S_{1} \cdots S_{n-1}\right\rangle .
$$

It remains to show that we can remove all relations apart from (R60-R70). Relations (R60-R66) contain defining relations for $\mathcal{P}_{n}=\langle\bar{s}, \bar{c}, \bar{e}, \bar{q}\rangle$ (see [20, Theorem 41]), so we may remove $c=S_{1} \cdots S_{n-1}$ and all relations from $(\mathrm{R} 44-\mathrm{R} 59)^{\prime}$ with no occurrence of the letter $o$. This leaves (R55-R59) and the relation $e=e o e$; the latter is part of (R61), so may be removed. Next note that (R60), (R67) and 
(R68) contain defining relations for $\mathcal{R}_{n}=\langle\bar{s}, \bar{c}, \bar{o}\rangle$ (see [70] or [27]), so we may remove all relations from (R55-R59)' with no occurrence of the letters $e, q$. This leaves (R58)', (R59)', and the relations

$$
o=o e o \quad \text { and } \quad o q=q o=o s o
$$

These last relations are already part of (R67) and (R68), so we are only left with (R58)' and (R59)'. The former is just (R70). For (R59)', first observe that $\bar{s}_{2} \bar{s}_{1} \bar{o} \bar{s}_{1} \bar{s}_{2}=\bar{c}^{2} \bar{o} \bar{c}^{n-2}$, so that $S_{2} S_{1} o S_{1} S_{2} \diamond$ $c^{2} o c^{n-2}$, by the above-mentioned fact that (R60-R70) contains defining relations for $\mathcal{R}_{n}=\langle\bar{s}, \bar{c}, \bar{o}\rangle$. Together with (R69), it then follows that $q S_{2} S_{1} o S_{1} S_{2} \diamond q c^{2} o c^{n-2} \diamond c^{2} o c^{n-2} q \diamond S_{2} S_{1} o S_{1} S_{2} q$.

Remark 4.13. The presentation in Theorem 4.12 includes defining relations for the symmetric group $\mathcal{S}_{n}$ (i.e., relations (R60)), plus a fixed (i.e., independent of $n$ ) number of extra relations: 26 such extra relations, to be precise. We make no claim that this is the minimal number of additional relations required.

We conclude this section by showing that the generating set in the previous result has the minimal possible size.

Theorem 4.14. For $n \geq 3, \operatorname{rank}\left(\mathcal{R} \mathcal{P}_{n}\right)=5$.

Proof. By Theorem 4.12, it suffices to show that any generating set for $\mathcal{R} \mathcal{P}_{n}$ has size at least 5. So suppose $\mathcal{R} \mathcal{P}_{n}=\langle\Sigma\rangle$. Since $\mathcal{R} \mathcal{P}_{n} \backslash \mathcal{S}_{n}$ is an ideal of $\mathcal{R} \mathcal{P}_{n}$, it follows that $\Sigma \cap \mathcal{S}_{n}$ is a generating set for $\mathcal{S}_{n}$. Since $\operatorname{rank}\left(\mathcal{S}_{n}\right)=2$, it follows that $\left|\Sigma \cap \mathcal{S}_{n}\right| \geq 2$. It therefore remains to show that $\left|\Sigma \backslash \mathcal{S}_{n}\right| \geq 3$.

Consider an expression $\bar{e}_{1}=\alpha_{1} \cdots \alpha_{k}$, where $\alpha_{1}, \ldots, \alpha_{k} \in \Sigma$. Let $1 \leq l \leq k$ be minimal so that $\alpha_{l} \notin \mathcal{S}_{n}$. Let $i=1\left(\alpha_{1} \cdots \alpha_{l-1}\right)$. Then

$$
\bar{e}_{i}=\left(\alpha_{1} \cdots \alpha_{l-1}\right)^{-1} \bar{e}_{1}\left(\alpha_{1} \cdots \alpha_{l-1}\right)=\alpha_{l}\left(\alpha_{l+1} \cdots \alpha_{k}\right)\left(\alpha_{1} \cdots \alpha_{l-1}\right)
$$

As in the proof of Theorem 3.15, it follows that $\alpha_{l}$ has domain $\{i\}^{c}$ and the block $\{i\}$. Similarly, it can be shown that $\Sigma \backslash \mathcal{S}_{n}$ contains: an element with domain $\{j\}^{c}$ and the rook dot $j$, for some $j \in[n]$; and an element with domain $[n]$ and non-trivial kernel.

Remark 4.15. The second paragraph of the previous proof works for $n=2$ as well, showing that $\operatorname{rank}\left(\mathcal{R} \mathcal{P}_{2}\right)=\operatorname{rank}\left(\mathcal{S}_{2}\right)+3=4$. It is easy to check that $\operatorname{rank}\left(\mathcal{R} \mathcal{P}_{n}\right)=1,3$ for $n=0,1$.

\section{Rook partition algebras}

Recall from [75] that the partition algebras are twisted semigroup algebras of the partition monoids. As noted in the introduction, this understanding has led to a rich flow of information between the theories of diagram semigroups and diagram algebras: see for example [15, 17, 20, 21, 24, 75]. In this section, we show how the results of previous sections lead to (algebra) presentations for the rook partition algebras and their singular ideals.

Let $F$ be a commutative ring with 1 , and let $S$ be a semigroup. Recall that a twisting from $S$ to $F$ is a map $\tau: S \times S \rightarrow F$ satisfying $\tau(a, b) \tau(a b, c)=\tau(a, b c) \tau(b, c)$ for all $a, b, c \in S$. Given such a twisting, the twisted semigroup algebra of $S$ over $F$ with respect to $\tau$, denoted $F^{\tau}[S]$, is defined to be the set of all finite formal $F$-linear combinations over $S$, with associative operation $\star$ defined on basis elements (and then extended linearly) by $a \star b=\tau(a, b) a b$ for each $a, b \in S$ (where $a b$ is the product 
of $a$ and $b$ in $S$ ). When the twisting is trivial (i.e., $\tau(a, b)=1 \in F$ for all $a, b \in S$ ), $F^{\tau}[S]=F[S]$ is just the (ordinary) semigroup algebra.

Given a fixed element $\delta \in F$, one may define a twisting from $\mathcal{R} \mathcal{P}_{n}$ to $F$ as follows. For $\alpha, \beta \in \mathcal{R} \mathcal{P}_{n}$, let $m(\alpha, \beta)$ denote the number of connected components in the product graph $\Gamma(\alpha, \beta)$ that involve only black vertices in the middle layer (i.e., double-dashed non-rook vertices). One may show that

$$
m(\alpha, \beta)+m(\alpha \beta, \gamma)=m(\alpha, \beta \gamma)+m(\beta, \gamma) \quad \text { for all } \alpha, \beta, \gamma \in \mathcal{R} \mathcal{P}_{n}
$$

As a result, one may then define a twisting

$$
\tau: \mathcal{R} \mathcal{P}_{n} \times \mathcal{R} \mathcal{P}_{n} \rightarrow F \quad \text { by } \quad \tau(\alpha, \beta)=\delta^{m(\alpha, \beta)} \quad \text { for all } \alpha, \beta \in \mathcal{R} \mathcal{P}_{n},
$$

and the resulting twisted semigroup algebra $F^{\tau}\left[\mathcal{R} \mathcal{P}_{n}\right]$ is called the rook partition algebra [33]. (The reason that white vertices (i.e., rook dots) do not figure in the count of $m(\alpha, \beta)$ is due to the exact nature of the above-mentioned embedding of $\mathcal{R} \mathcal{P}_{n}$ in $\mathcal{P}_{n+1}$; see [33 for more details.) Note also that $\tau$ restricts to a twisting from $\mathcal{R} \mathcal{P}_{n} \backslash \mathcal{S}_{n}$ to $F$, so we may consider the singular rook partition algebra, $F^{\tau}\left[\mathcal{R} \mathcal{P}_{n} \backslash \mathcal{S}_{n}\right]$.

A general result from [20] shows how a (monoid or semigroup) presentation for $S$ leads to an (algebra) presentation for $F^{\tau}[S]$ in the case that the image of the twisting $\tau$ lies in $G(F)$, the group of units of $F$. The reader is referred to [20, Section 6] for full details. The next result follows immediately from [20, Theorem 44] and earlier results of the current paper (as stated below).

Theorem 5.1. Suppose $F$ is a commutative ring with identity, and let $\delta \in G(F)$ be a unit in $F$.

(i) An algebra presentation for the rook partition algebra, $F^{\tau}\left[\mathcal{R} \mathcal{P}_{n}\right]$, may be obtained from any of the above monoid presentations for $\mathcal{R} \mathcal{P}_{n}$ by:

(a) changing the relations $e_{i}^{2}=e_{i}$ to $e_{i}^{2}=\delta e_{i}$ in Theorem 4.7; or

(b) changing the relations $e^{2}=e$ to $e^{2}=\delta e$ in Theorem 4.11 or Theorem 4.12.

(ii) An algebra presentation for the singular rook partition algebra, $F^{\tau}\left[\mathcal{R} \mathcal{P}_{n} \backslash \mathcal{S}_{n}\right]$, may be obtained from the semigroup presentation for $\mathcal{R} \mathcal{P}_{n} \backslash \mathcal{S}_{n}$ in Theorem 3.14 by changing the relations $e_{i}^{2}=e_{i}$ to $e_{i}^{2}=\delta e_{i}$.

\section{References}

[1] K. Auinger, Yuzhu Chen, Xun Hu, Yanfeng Luo, and M. V. Volkov. The finite basis problem for Kauffman monoids. Algebra Universalis, 74(3-4):333-350, 2015.

[2] Karl Auinger. Pseudovarieties generated by Brauer type monoids. Forum Math., 26(1):1-24, 2014.

[3] Karl Auinger, Igor Dolinka, and Mikhail V. Volkov. Equational theories of semigroups with involution. J. Algebra, $369: 203-225,2012$.

[4] Karl Auinger, Igor Dolinka, and Mikhail V. Volkov. Matrix identities involving multiplication and transposition. J. Eur. Math. Soc. (JEMS), 14(3):937-969, 2012.

[5] Georgia Benkart and Tom Halverson. Motzkin algebras. European J. Combin., 36:473-502, 2014.

[6] Matthew Bloss. G-colored partition algebras as centralizer algebras of wreath products. J. Algebra, 265(2):690$710,2003$.

[7] Mirjana Borisavljević, Kosta Došen, and Zoran Petrić. Kauffman monoids. J. Knot Theory Ramifications, 11(2):127-143, 2002.

[8] Richard Brauer. On algebras which are connected with the semisimple continuous groups. Ann. of Math. (2), 38(4):857-872, 1937.

[9] Peter J. Cameron. Permutation groups, volume 45 of London Mathematical Society Student Texts. Cambridge University Press, Cambridge, 1999.

[10] Anton Cox, John Graham, and Paul Martin. The blob algebra in positive characteristic. J. Algebra, 266(2):584$635,2003$. 
[11] Zajj Daugherty and Rosa Orellana. The quasi-partition algebra. J. Algebra, 404:124-151, 2014.

[12] Momar Dieng, Tom Halverson, and Vahe Poladian. Character formulas for $q$-rook monoid algebras. J. Algebraic Combin., 17(2):99-123, 2003.

[13] Igor Dolinka and James East. Twisted Brauer monoids. Preprint, 2015, arXiv:1510.08666.

[14] Igor Dolinka and James East. The idempotent generated subsemigroup of the Kauffman monoid. Preprint, 2016, arXiv:1602.01157.

[15] Igor Dolinka, James East, Athanasios Evangelou, Des FitzGerald, Nicholas Ham, James Hyde, and Nicholas Loughlin. Enumeration of idempotents in diagram semigroups and algebras. J. Combin. Theory Ser. A, 131:119152, 2015.

[16] Igor Dolinka, James East, Athanasios Evangelou, Des FitzGerald, Nicholas Ham, James Hyde, and Nicholas Loughlin. Idempotent statistics of the Motzkin and Jones monoids. Preprint, 2015, arXiv:1507.04838.

[17] Igor Dolinka, James East, and Robert D. Gray. Motzkin monoids and partial Brauer monoids. Preprint, 2015, arXiv: 1512.02279.

[18] James East. Cellular algebras and inverse semigroups. J. Algebra, 296(2):505-519, 2006.

[19] James East. Braids and order-preserving partial permutations. J. Knot Theory Ramifications, 19(8):1025-1049, 2010.

[20] James East. Generators and relations for partition monoids and algebras. J. Algebra, 339:1-26, 2011.

[21] James East. On the singular part of the partition monoid. Internat. J. Algebra Comput., 21(1-2):147-178, 2011.

[22] James East. A symmetrical presentation for the singular part of the symmetric inverse monoid. Algebra Universalis, 74(3-4):207-228, 2015.

[23] James East and D. G. FitzGerald. The semigroup generated by the idempotents of a partition monoid. J. Algebra, 372:108-133, 2012.

[24] James East and R. D. Gray. Diagram monoids and Graham-Houghton graphs: idempotents and generating sets of ideals. Preprint, 2014, arXiv: 1404.2359.

[25] John Enyang. Jucys-Murphy elements and a presentation for partition algebras. J. Algebraic Combin., 37(3):401454, 2013.

[26] John Enyang. A seminormal form for partition algebras. J. Combin. Theory Ser. A, 120(7):1737-1785, 2013.

[27] Vítor H. Fernandes. Presentations for some monoids of partial transformations on a finite chain: a survey. In Semigroups, algorithms, automata and languages (Coimbra, 2001), pages 363-378. World Sci. Publ., River Edge, NJ, 2002.

[28] D. G. FitzGerald and Kwok Wai Lau. On the partition monoid and some related semigroups. Bull. Aust. Math. Soc., 83(2):273-288, 2011.

[29] D. G. FitzGerald and Jonathan Leech. Dual symmetric inverse monoids and representation theory. J. Austral. Math. Soc. Ser. A, 64(3):345-367, 1998.

[30] N. D. Gilbert. Presentations of the inverse braid monoid. J. Knot Theory Ramifications, 15(5):571-588, 2006.

[31] J. J. Graham and G. I. Lehrer. Cellular algebras. Invent. Math., 123(1):1-34, 1996.

[32] Cheryl Grood. A Specht module analog for the rook monoid. Electron. J. Combin., 9(1):Research Paper 2, 10 pp. (electronic), 2002.

[33] Cheryl Grood. The rook partition algebra. J. Combin. Theory Ser. A, 113(2):325-351, 2006.

[34] Tom Halverson. Representations of the q-rook monoid. J. Algebra, 273(1):227-251, 2004.

[35] Tom Halverson and Elise delMas. Representations of the Rook-Brauer algebra. Comm. Algebra, 42(1):423-443, 2014.

[36] Tom Halverson and Arun Ram. q-rook monoid algebras, Hecke algebras, and Schur-Weyl duality. Zap. Nauchn. Sem. S.-Peterburg. Otdel. Mat. Inst. Steklov. (POMI), 283(Teor. Predst. Din. Sist. Komb. i Algoritm. Metody. 6):224-250, 262-263, 2001.

[37] Tom Halverson and Arun Ram. Partition algebras. European J. Combin., 26(6):869-921, 2005.

[38] Peter M. Higgins. Techniques of semigroup theory. Oxford Science Publications. The Clarendon Press, Oxford University Press, New York, 1992.

[39] J. M. Howie. The subsemigroup generated by the idempotents of a full transformation semigroup. J. London Math. Soc., 41:707-716, 1966.

[40] J. M. Howie. Idempotent generators in finite full transformation semigroups. Proc. Roy. Soc. Edinburgh Sect. A, 81(3-4):317-323, 1978.

[41] John M. Howie. Fundamentals of semigroup theory, volume 12 of London Mathematical Society Monographs. New Series. The Clarendon Press, Oxford University Press, New York, 1995. Oxford Science Publications.

[42] V. F. R. Jones. Hecke algebra representations of braid groups and link polynomials. Ann. of Math. (2), 126(2):335388, 1987.

[43] V. F. R. Jones. The Potts model and the symmetric group. In Subfactors (Kyuzeso, 1993), pages 259-267. World Sci. Publ., River Edge, NJ, 1994. 
[44] Louis H. Kauffman. An invariant of regular isotopy. Trans. Amer. Math. Soc., 318(2):417-471, 1990.

[45] A. Joseph Kennedy. Class partition algebras as centralizer algebras of wreath products. Comm. Algebra, $35(1): 145-170,2007$.

[46] A. Joseph Kennedy and G. Muniasamy. Rook version of colored partition algebras. Bull. Math. Sci., 3(1):1-17, 2013.

[47] Ganna Kudryavtseva and Victor Maltcev. Two generalisations of the symmetric inverse semigroups. Publ. Math. Debrecen, 78(2):253-282, 2011.

[48] Ganna Kudryavtseva, Victor Maltcev, and Abdullahi Umar. Presentation for the partial dual symmetric inverse monoid. Comm. Algebra, 43(4):1621-1639, 2015.

[49] Ganna Kudryavtseva and Volodymyr Mazorchuk. On presentations of Brauer-type monoids. Cent. Eur. J. Math., 4(3):413-434 (electronic), 2006.

[50] Ganna Kudryavtseva and Volodymyr Mazorchuk. Schur-Weyl dualities for symmetric inverse semigroups. J. Pure Appl. Algebra, 212(8):1987-1995, 2008.

[51] Kwok Wai Lau and D. G. FitzGerald. Ideal structure of the Kauffman and related monoids. Comm. Algebra, 34(7):2617-2629, 2006.

[52] Mark V. Lawson. Inverse semigroups. World Scientific Publishing Co., Inc., River Edge, NJ, 1998. The theory of partial symmetries.

[53] Gustav Lehrer and R. B. Zhang. The Brauer category and invariant theory. J. Eur. Math. Soc. (JEMS), $17(9): 2311-2351,2015$.

[54] Gustav Lehrer and Ruibin Zhang. The second fundamental theorem of invariant theory for the orthogonal group. Ann. of Math. (2), 176(3):2031-2054, 2012.

[55] Stephen Lipscomb. Symmetric inverse semigroups, volume 46 of Mathematical Surveys and Monographs. American Mathematical Society, Providence, RI, 1996.

[56] Victor Maltcev and Volodymyr Mazorchuk. Presentation of the singular part of the Brauer monoid. Math. Bohem., 132(3):297-323, 2007.

[57] P. P. Martin. The partition algebra and the Potts model transfer matrix spectrum in high dimensions. J. Phys. A, 33(19):3669-3695, 2000.

[58] Paul Martin. Temperley-Lieb algebras for nonplanar statistical mechanics - the partition algebra construction. J. Knot Theory Ramifications, 3(1):51-82, 1994.

[59] Paul Martin. The structure of the partition algebras. J. Algebra, 183(2):319-358, 1996.

[60] Paul Martin and Volodymyr Mazorchuk. On the representation theory of partial Brauer algebras. Q. J. Math., 65(1):225-247, 2014.

[61] Paul Martin and Hubert Saleur. The blob algebra and the periodic Temperley-Lieb algebra. Lett. Math. Phys., 30(3):189-206, 1994.

[62] Paul P. Martin and David Woodcock. On the structure of the blob algebra. J. Algebra, 225(2):957-988, 2000.

[63] Volodymyr Mazorchuk. On the structure of Brauer semigroup and its partial analogue. Problems in Algebra, 13:29-45, 1998.

[64] Volodymyr Mazorchuk. Endomorphisms of $\mathfrak{B}_{n}, \mathcal{P} \mathfrak{B}_{n}$, and $\mathfrak{C}_{n}$. Comm. Algebra, 30(7):3489-3513, 2002.

[65] Eliakim Hastings Moore. Concerning the abstract groups of order $k$ ! and $\frac{1}{2} k$ ! holohedrically isomorphic with the symmetric and the alternating substitution-groups on $k$ letters. Proc. London Math. Soc., 28(1):357-366, 1897.

[66] T. E. Nordahl and H. E. Scheiblich. Regular *-semigroups. Semigroup Forum, 16(3):369-377, 1978.

[67] Rosa C. Orellana. On partition algebras for complex reflection groups. J. Algebra, 313(2):590-616, 2007.

[68] Rowena Paget. Representation theory of q-rook monoid algebras. J. Algebraic Combin., 24(3):239-252, 2006.

[69] M. Parvathi and A. Joseph Kennedy. $G$-vertex colored partition algebras as centralizer algebras of direct products. Comm. Algebra, 32(11):4337-4361, 2004.

[70] L. M. Popova. Defining relations in some semigroups of partial transformations of a finite set (in Russian). Uchenye Zap. Leningrad Gos. Ped. Inst., 218:191-212, 1961.

[71] Eliezer Posner, Kris Hatch, and Megan Ly. Presentation of the Motzkin monoid. Preprint, 2013, arXiv: 1301.4518.

[72] Louis Solomon. Representations of the rook monoid. J. Algebra, 256(2):309-342, 2002.

[73] H. N. V. Temperley and E. H. Lieb. Relations between the "percolation" and "colouring" problem and other graphtheoretical problems associated with regular planar lattices: some exact results for the "percolation" problem. Proc. Roy. Soc. London Ser. A, 322(1549):251-280, 1971.

[74] Hermann Weyl. The classical groups. Princeton Landmarks in Mathematics. Princeton University Press, Princeton, NJ, 1997. Their invariants and representations, Fifteenth printing, Princeton Paperbacks.

[75] Stewart Wilcox. Cellularity of diagram algebras as twisted semigroup algebras. J. Algebra, 309(1):10-31, 2007. 